\title{
A systematic review of evidence to inform HIV prevention interventions among men who have sex with men in Europe
}

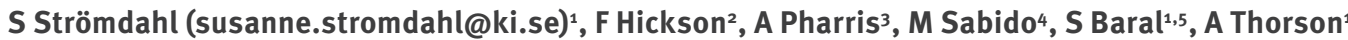

1. Department of Public Health Sciences, Karolinska Institutet, Sweden

2. Sigma Research, Department of Social \& Environmental Health Research, London School of Hygiene \& Tropical Medicine, United Kingdom

3. European Centre for Disease Prevention and Control (ECDC), Stockholm, Sweden

4. Fundació Sida i Societat, Barcelona, Spain

5. Department of Epidemiology, Johns Hopkins Bloomberg School of Public Health, Baltimore, USA

Strömdahl S, Hickson F, Pharris A, Sabido M, Baral S, Thorson A. A systematic review of evidence to inform HIV prevention interventions among men who have sex with men in Europe. Euro Surveill. 2015;20(15):pii=21096. Available online: http://www.eurosurveillance.org/ViewArticle.aspx?Articleld=21096

An estimated $42 \%$ of all newly diagnosed HIV cases in Europe in 2013 were transmitted during sex between men. This review was performed to identify and describe studies evaluating the efficacy and effectiveness of HIV prevention interventions among men who have sex with men (MSM), in relation to implementation data from European settings. A systematic search was performed individually for 24 interventions. Data were extracted from studies including efficacy or implementation data from European settings, appraised for efficacy, implementation and plausibility, and assigned a grade (1-4) according to the Highest Attainable Standard of Evidence (HASTE) framework. Four interventions (condom use, peer outreach, peer-led groups, and using universal coverage of antiretroviral treatment and treatment as prevention) were assigned the highest HASTE grade, 1. Another four interventions were assigned $2 a$ for probable recommendation, including voluntary counselling and testing for HIV, using condom-compatible lubricant, using post-exposure prophylaxis, and individual counselling for MSM living with HIV. In addition, seven interventions were assigned a grade of $2 \mathrm{~b}$, for possible recommendation. Encouragingly, 15 interventions were graded to be strongly, probably or possibly recommended. In the relatively resource-rich European setting, there is an opportunity to provide global leadership with regard to the regional scale-up of comprehensive HIV prevention interventions for MSM.

\section{Introduction}

In 2012 the global burden of HIV was estimated to include 35.3 million people living with the virus (people living with HIV, PLWH). Among adults between the ages of 15-49 years old HIV prevalence was estimated at $0.8 \%$ [1]. Globally there is a declining trend in new infections, morbidity and mortality due to HIV/AIDS [2].
Improved treatment regimens and access to treatment are important factors behind these trends [2].

Gay, bisexual and other men who have sex with men (MSM) are disproportionately affected by HIV in every setting where data are available [3]. HIV rates reported among MSM show an increasing trend, in contrast to the declining trends reported in the general population [4]. A 2013 systematic review of HIV epidemiology in 33 high-income countries where data were available estimated a total of 2.3 million PLWH [5] and a malefemale median case ratio of $2.5: 1[5,6]$, indicating male-predominant epidemics. High-income countries where antiretroviral treatment (ART) and prevention services are available show increasing trends in HIV prevalence among MSM $[5,7]$.

Of the 29,157 persons diagnosed with HIV and reported in the European Union/European Economic area (EU/ EEA) in $2013,42 \%$ of cases were estimated to be due to sex between men [8]. Since 2006, MSM represent the only key population where an increase in HIV diagnoses has been observed, with a $33 \%$ increase between 2004 and 2013 in the EU/EEA overall and with increases of more than $100 \%$ observed in some EU countries during the past decade, including Bulgaria, Cyprus, Czech Republic, Hungary, Romania, and Slovakia [9]. HIV prevalence among MSM was estimated to be at or above $5 \%$ in 14 of the 26 EU/EEA countries reporting national data in 2012 [10].

In accounting for the relatively higher rates of HIV among MSM compared with the general population, recent epidemic modelling highlights the importance of the higher transmissibility of HIV during unprotected anal intercourse (as opposed to vaginal) and the importance of insertive/receptive sexual role versatility among MSM $[4,11]$. Clusters of HIV transmission 
indicative of outbreaks within sexual networks of MSM may also play an important role in the higher transmission probability reported [12-14].

The current picture of the HIV epidemic among MSM in Europe highlights significant variation between countries. Biological and behavioural surveillance systems vary across European countries, as do the extent of sexual health needs assessment, collaborative service planning and the availability of acceptable and accessible sexual health services [15]. Prevalence data for MSM, a population of unknown size, can be estimated in diverse ways and therefore prevalence rates may not be fully comparable between countries. Most European countries report the number of newly diagnosed cases annually [10].

Community, research, medical and public health efforts to prevent HIV have existed in Europe for over three decades, with European gay community organisations at the forefront of peer-led HIV prevention globally. However, overall national responses have been inadequate to contain HIV epidemics among MSM, with continuing high and in some countries increasing HIV incidence among MSM $[5,16]$.

HIV prevention interventions for MSM are purposeful activities intended to increase the uptake of HIV precautionary behaviours or to reduce HIV risk behaviours. Intervention activities can target MSM directly, they can be directed to intermediaries who deliver activities to MSM, or they can influence the policy and service environment. The effectiveness of HIV prevention interventions among MSM has been assessed previously, most recently by the World Health Organization Global Guidelines process in 2010-11 [17]. In order to capture more recent data in the rapidly evolving field of HIV prevention and to ensure context-specific relevance, there was a need to update and extend the previous reviews and catalogue the evidence in order to inform MSM prevention interventions in Europe $[18,19]$.

The objectives of this review were to identify and describe studies evaluating the efficacy, and effectiveness of HIV prevention interventions among MSM in relation to implementation data from the European setting, and to further appraise the evidence according to the Highest Attainable Standard of Evidence (HASTE) framework [20]. The review of evidence was performed in order to inform the development of guidance by the European Centre for Disease Control and Prevention (ECDC) to Member States on the commissioning and delivery of HIV prevention interventions to MSM in the EU/EEA [21].

\section{Methods}

In this review the term 'men who have sex with men' (MSM) refers to the population of men engaged in same-sex sexual behaviour, inclusive of sexual identities (e.g. gay, bisexual, straight, experimenting, etc.)

\section{FIGURE}

Work process for systematic review of HIV prevention interventions among men who have sex with men within the European setting, searches performed December 2012-February 2013

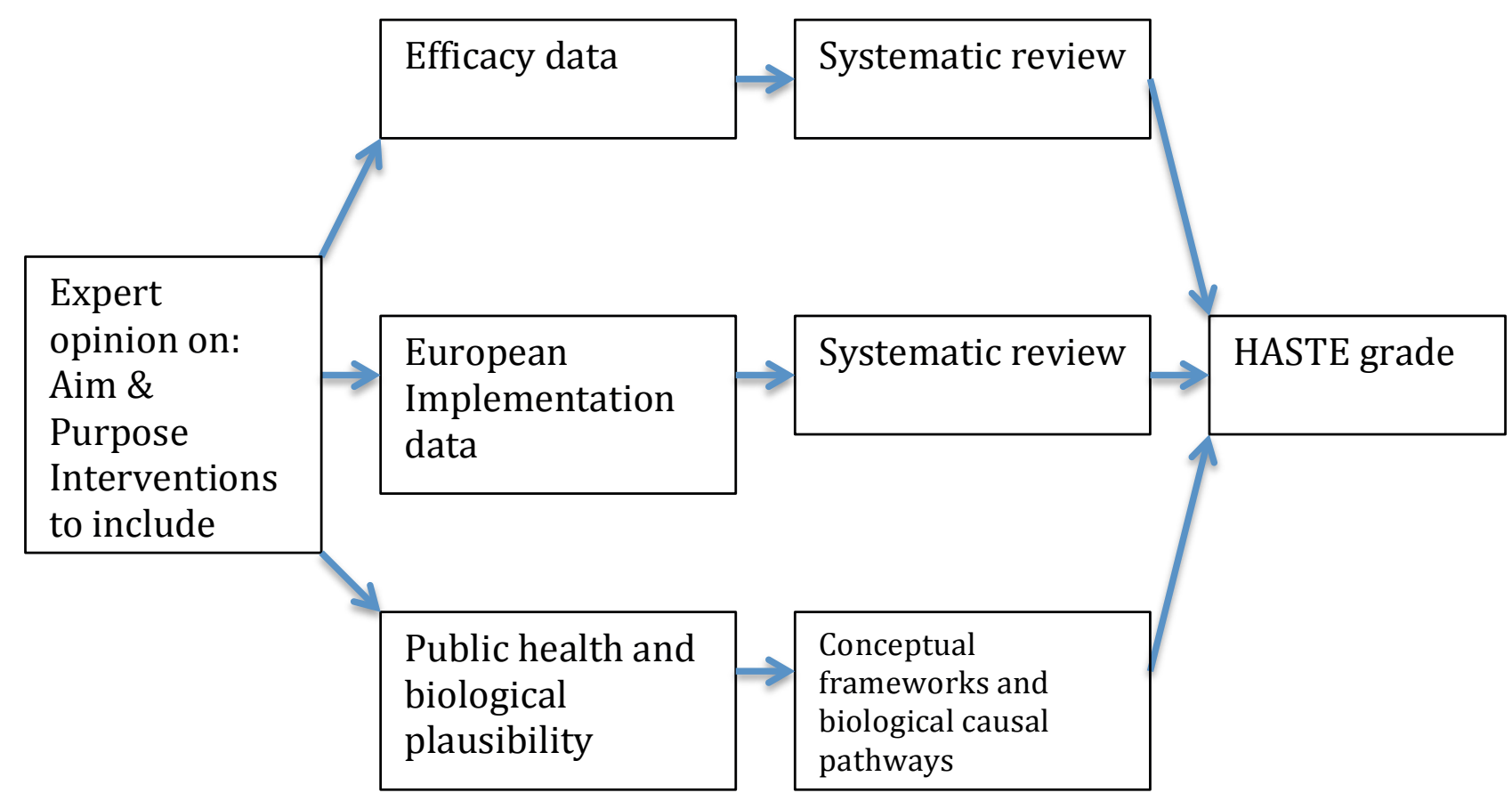


TABLE 1

Highest Attainable Standard of Evidence (HASTE) system for HIV interventions ${ }^{\mathrm{a}}$

\begin{tabular}{|c|c|c|c|}
\hline \multicolumn{2}{|l|}{ Grade level } & Strength of recommendation & Explanation \\
\hline \multicolumn{2}{|l|}{ Grade 1} & Strong & $\begin{array}{l}\text { - High plausibility } \\
\text { - Efficacy is consistent } \\
\text { - Large body of consistent implementation data }\end{array}$ \\
\hline \multirow{3}{*}{ Grade 2} & Grade $2 a$ & Conditional: probable & $\begin{array}{l}\text { - Plausibility } \\
\text { - Limited efficacy data } \\
\text { - Consistently effective from implementation data }\end{array}$ \\
\hline & Grade $2 b$ & Conditional: possible & $\begin{array}{l}\text { - Plausibility } \\
\text { - Limited or inconsistent efficacy data } \\
\text { - Limited or paucity of implementation data }\end{array}$ \\
\hline & Grade $2 c$ & Conditional: pending & $\begin{array}{l}\text { - Plausibility } \\
\text { - Ongoing efficacy trials }\end{array}$ \\
\hline \multicolumn{2}{|l|}{ Grade 3} & Insufficient & $\begin{array}{l}\text { - Undefined plausibility } \\
\text { - Inconsistent data } \\
\text { - Inconsistent or paucity of implementation data }\end{array}$ \\
\hline \multicolumn{2}{|l|}{ Grade 4} & Inappropriate & $\begin{array}{l}\text { - Consistent data demonstrating lack of efficacy } \\
\text { - Consensus from implementation data of inappropriate intervention }\end{array}$ \\
\hline
\end{tabular}

and sexual desire. The term MSM includes people who identify as men, and therefore includes transgender men who have sex with men. Transgender women might share some biological risks with MSM such as receptive anal intercourse, but recent data shows a higher HIV burden in this group, indicating a different epidemic scenario [22], and therefore transgender women are not included as a sub-group of MSM in this review. We use the term MSM in this review recognising the diversity and heterogeneity of this group but also the limitations of this term.

First, we made a comprehensive list of known interventions that address primary HIV transmission among MSM, inclusive of biomedical, psychosocial, and programmatic interventions. The list was developed, discussed and agreed by an expert review group and included medical, social science and policy experts, programme implementers from non-governmental organisations and government representatives. The group was convened by the ECDC for the development of European guidelines on HIV prevention in MSM. A systematic review was performed for each intervention included (Figure 1).

Existing evidence from randomised controlled trials (RCTs) evaluating public health interventions with biological endpoints for MSM populations are limited, which highlights the need for strategies additional to the Grading of Recommendations Assessment, Development and Evaluation (GRADE) system when performing a review such as this $[18,19,23]$. The HASTE system builds on the GRADE system and was developed specifically to evaluate evidence regarding HIV/ sexually transmitted infection (STI) interventions among most at-risk populations, in particular MSM
[19]. HASTE takes into account three categories that are given equal weight: efficacy data, implementation science data and biological and public health plausibility (Table 1) [19]. Hill's criteria for causality remain the most relevant set of determinants of whether an intervention causes prevention and/or mitigation of disease in the HASTE grading system [24].

Implementation data were defined as data reporting on availability, acceptability, uptake, feasibility of implementation, implementation costs, and effectiveness of the intervention among MSM in the European setting.

Public health plausibility was defined as the intervention having a likely pathway leading to a decrease in HIV incidence. For example, HIV testing itself might not lead directly to lower HIV incidence, but it has a crucial role because it is on the pathway to uptake of ART for people living with HIV, which does decrease HIV onward transmission and HIV-related morbidity and mortality.

\section{Search strategies}

The Population, Intervention, Comparison, Outcome (PICO) model was used to develop inclusion criteria and search terms per intervention [25]. The population for the intervention was MSM. All types of comparison and no comparison were included. Outcomes included were biological markers (prevalence and incidence of HIV/STIS), self-reported diagnoses of HIV infection and self-reported behavioural outcomes on condom use or unprotected anal intercourse (UAI). Studies reporting on implementation data were only included if performed in Europe. Systematic reviews previously performed on these topics were included. Non-peerreviewed literature was not used as a source of original 
TABLE 2

Number of articles found through search strategies, screened and included according to inclusion criteria for HIV prevention interventions among men who have sex with men in Europe

\begin{tabular}{|c|c|c|c|}
\hline Interventions & $\begin{array}{l}\text { Articles } \\
\text { identified } \\
\text { through } \\
\text { searches }\end{array}$ & $\begin{array}{l}\text { Number of } \\
\text { articles } \\
\text { included } \\
\text { addressing } \\
\text { efficacy }\end{array}$ & $\begin{array}{l}\text { Number of } \\
\text { articles } \\
\text { included } \\
\text { addressing } \\
\text { implementation }\end{array}$ \\
\hline Condom use & 130 & 1 & 3 \\
\hline Universal coverage of antiretroviral treatment and treatment as prevention & 9 & 1 & 4 \\
\hline Peer-led group interventions & 326 & 2 & o \\
\hline Peer outreach & 326 & 2 & o \\
\hline Voluntary HIV counselling and testing & 717 & 2 & 8 \\
\hline Condom-compatible lubricant use (when using condoms) & 130 & 5 & 2 \\
\hline Post-exposure prophylaxis & 28 & 3 & 2 \\
\hline Individual counselling for MSM living with HIV & 327 & 4 & 2 \\
\hline Peer-led group interventions targeting MSM living with HIV & 326 & 1 & 2 \\
\hline Sex venue-based interventions & 25 & 1 & 8 \\
\hline Social marketing interventions & 476 & 3 & 7 \\
\hline Individual counselling for MSM & 327 & 2 & 1 \\
\hline Internet-based HIV prevention messages & 40 & 6 & 4 \\
\hline Training for healthcare providers to provide comprehensive care for MSM & 225 & o & 1 \\
\hline MSM friendly clinics & 234 & o & 1 \\
\hline Voluntary anonymous partner notification & 126 & 0 & 7 \\
\hline Voluntary medical male circumcision & 49 & 3 & 2 \\
\hline Pre-exposure prophylaxis & 4 & 1 & o \\
\hline Campaigns for lesbian, gay, bisexual and trans equality & 3 & 0 & 1 \\
\hline Female condom use & 4 & 3 & 1 \\
\hline Serosorting & 9 & 3 & 4 \\
\hline Avoid ejaculation of semen orally & 226 & 3 & o \\
\hline Avoiding poppers during anal intercourse & 5 & 0 & 0 \\
\hline Reducing alcohol binge drinking among MSM & 119 & 1 & 0 \\
\hline Total number of articles ${ }^{a}$ & 3,865 & 47 & 60 \\
\hline
\end{tabular}

MSM: Men who have sex with men.

Searches were performed between 10 December 2012 and 8 February 2013.

a Search strategies captured the same articles to some extent.

data, but these documents did guide further searches for literature. Studies published in English, French, and Spanish were included. Studies not fitting these criteria were excluded from the review.

Electronic searches were performed in PubMed, Embase, Medline, Cinahl, PsycINFO, the Cochrane Library and the World Health Organization publication database. The search included medical subject headings (MeSH) terms for HIV or AIDS, and terms associated with MSM and the specific interventions reviewed (Annex I). Searches were particularly designed to be broad and comprehensive initially and were performed between 10 December 2012 and 8 February 2013. We reviewed the search strategies performed between 8 June 2010 and 17 March 2011 to guide the WHO's 2011 recommendations for 'Prevention and treatment of HIV and other sexually transmitted infections among MSM and transgender people', and where relevant these were updated up to 8 February 2013 [17].

\section{Screening and data extraction}

After the removal of duplicates, titles were screened independently by two researchers (SS, MS) to exclude those that did not fit the inclusion criteria. When a title was judged to be relevant, the abstract was reviewed and included if the inclusion criteria were met. When it was not clear whether the abstract met the inclusion criteria, the full article was reviewed.

For all selected articles, data were extracted by two researchers (SS, MS) using a pre-designed data extraction form that included details on individual study design, methods of recruitment, sampling frame, 
sample size, location, response rate, analysis performed, results, confounders, reported HIV prevalence/incidence and self-reported sexual behaviour, HIV prevalence/incidence and self-reported sexual behaviour in comparison groups (if provided).

\section{Analysis}

First, a critical appraisal of the quality of each individual efficacy study was performed by two researchers (SS, MS) using a checklist approach to assess the methodological components [26]. In the next step a compilation was done, including all relevant studies or reviews for each intervention. The data compilations were then reviewed by SS and MS, together with a senior researcher (AT), in order to check for consistency. Implementation studies were appraised for availability, acceptability, uptake, feasibility of implementation, implementation costs, and (when available) effectiveness of the intervention among MSM in the European setting.

A paucity of implementation data was found in the EU/ EEA setting. Therefore the HASTE grading framework was adjusted slightly regarding grade $2 \mathrm{~b}$. Interventions with limited efficacy data, defined as being plausible but lacking European implementation data were assigned a grade $2 \mathrm{~b}$. Interventions without established efficacy were assigned a grade $2 c$ in order to differentiate interventions with (grade $2 \mathrm{~b}$ ) and without (grade 2c) established efficacy.

The evidence gathered for each intervention was reviewed using the HASTE grading framework [20]. The grading was performed independently by two researchers (SS and MS) and showed high agreement (90\%). All grades were reviewed by a senior scientist (AT) and discrepancies were discussed initially in the smaller group, and following that in a conference with the co-authors (SS, MS, AP, FH, SB, AT) where remaining discrepancies and questions were resolved. Biological and public health plausibility was determined through a process of discussions within the team of co-authors.

\section{Results}

Twenty-four HIV prevention interventions for MSM were included and reviewed. Table 2 presents the intervention topics as well as the number of articles found through search strategies, screened and included per intervention.

\section{Interventions assigned a strong} recommendation (HASTE grade 1)

Four interventions were assigned a HASTE grade 1: condom use, universal coverage of antiretroviral treatment and treatment as prevention, peer-led group interventions and peer outreach within the MSM community.

\section{Condom use}

Consistent efficacy data showed that condom use during anal intercourse prevents HIV transmission. A systematic review including five cohort studies $(n=8,825)$ reported that condom use reduced HIV transmission (relative risk (RR): 0.36; 95\% confidence interval (CI) 0.20-0.67) [27-32]. Implementation data supported acceptability and feasibility of condom use among MSM and the feasibility of condom distribution programmes in Europe [33-35]. Thirteen per cent of MSM in European countries reported they had UAI in the last 12 months solely because they did not have a condom available, which points towards an unmet need of condoms among some MSM [36]. Plausibility was determined as condoms are a barrier method, thereby preventing the transmission of HIV. No serious potential risk with using condoms was identified.

\section{Universal coverage of antiretroviral treatment and treatment as prevention}

A randomised, double-blinded controlled trial with 1,763 serodiscordant heterosexual couples and 37 serodiscordant male MSM couples, reported a relative reduction of $96 \%$ in the number of linked HIV-1 transmissions cases resulting from the early initiation of antiretroviral therapy, as compared with delayed therapy. Since only 37 MSM couples were included, the size of the relative reduction reported may not accurately reflect the protective effect on sexual transmission between MSM. Implementation data reports that ART programmes are available in all EU/EEA countries. However, national treatment guidelines show diversity regarding when to start treatment (at diagnosis or at $\mathrm{CD}_{4}$ count threshold level) [10]. Plausibility was deemed high as ART decreases the replication of HIV-1 and has been shown to reduce the amount of HIV-1 in genital secretions [37], which is likely to be the mechanism by which antiretroviral treatment reduces sexual transmission of the virus among MSM. However, the effectiveness of this intervention is dependent on comprehensive HIV testing programmes among MSM, and effective linkage to and retention in high-quality HIV treatment and care.

\section{Peer-led group interventions}

Peer-led group interventions, defined as interactive group activities where a trained peer facilitates promotion of precautionary behaviours for HIV, were found to cause a significant reduction in UAI by a systematic review including 21 studies $(n=5,197$ and one study of unknown sample size) [38]. The size of the reduction ranged from $13 \%$ to $33 \%$ [38-40]. Implementation data show high uptake of peer-led group interventions among MSM in Europe [38,41]. The intervention was judged plausible as the effect of peer-led group interventions may decrease high-risk behaviours for HIV through a combination of increased knowledge, social learning, influence of peers and normative group behaviour [38].

\section{Peer-outreach}

A review of systematic reviews that included 4 reviews (in total including 11 studies with $n>7,890$ ) found that peer outreach interventions, where a trained peer approaches MSM in community settings providing 
Highest Attainable Standard of Evidence (HASTE) evaluation per HIV prevention intervention in men who have sex with men in Europe

\begin{tabular}{|c|c|c|c|c|c|}
\hline \multirow[b]{2}{*}{ Intervention } & \multirow[b]{2}{*}{ Outcome } & Efficacy data & Implementation data & Plausibility & Grading \\
\hline & & Consistent/limited/inconsistent/NA & $\begin{array}{l}\text { Available/NA from } \\
\text { European setting }\end{array}$ & $\begin{array}{l}\text { Biological plausibility / Public } \\
\text { health plausibity }\end{array}$ & $\begin{array}{l}\text { HASTE } \\
\text { grade } 1-4\end{array}$ \\
\hline Condom use & $\begin{array}{l}\text { HIV } \\
\text { incidence }\end{array}$ & $\begin{array}{l}\text { Efficacy data are consistent. A } \\
\text { systematic review from } 2010 \text { including } \\
\text { five cohort studies }(n=8,825) \text { reports } \\
\text { that the overall effect of condom } \\
\text { use on HIV transmission was RR: } \\
0.36 ; 95 \% \mathrm{Cl} 0.20-0.67, \text { consistent } \\
\text { condom use was found to reduce HIV } \\
\text { transmission by } 64 \%[27-32] \text {. }\end{array}$ & $\begin{array}{l}\text { Available. } \\
\text { Distribution of } \\
\text { condoms is feasible. } \\
\text { High acceptability } \\
\text { and feasibility of } \\
\text { condom use has been } \\
\text { reported among MSM } \\
\text { [33-35] }\end{array}$ & $\begin{array}{l}\text { The intervention has biological } \\
\text { plausibility. The condom acts as } \\
\text { barrier, thereby preventing the } \\
\text { transmission of HIV. } \\
\text { No serious potential risk with } \\
\text { using condoms has been } \\
\text { identified. Operations research } \\
\text { emphasises the importance of } \\
\text { condom-compatible lubricant use } \\
\text { at condom use during anal sex } \\
\text { [120]. }\end{array}$ & $\begin{array}{l}\text { Strong, } \\
\text { grade } 1\end{array}$ \\
\hline $\begin{array}{l}\text { Universal } \\
\text { coverage of } \\
\text { antiretroviral } \\
\text { treatment } \\
\text { and } \\
\text { treatment as } \\
\text { prevention }\end{array}$ & $\begin{array}{l}\text { HIV } \\
\text { incidence }\end{array}$ & $\begin{array}{l}\text { Efficacy is consistent [37, 121- } \\
\text { 124]. A randomised, double- } \\
\text { blinded controlled trial with } 1,763 \\
\text { serodiscordant heterosexual couples } \\
\text { and } 37 \text { serodiscordant male MSM } \\
\text { couples, reported a relative reduction } \\
\text { of } 96 \% \text { in the number of linked HIV-1 } \\
\text { transmission cases resulting from the } \\
\text { early initiation of ART, as compared } \\
\text { with delayed therapy [37]. }\end{array}$ & $\begin{array}{l}\text { Available. } \\
\text { Implementation } \\
\text { data reports that } \\
\text { ART programmes } \\
\text { are available in all } \\
\text { EU/EEA countries. } \\
\text { However, national } \\
\text { treatment guidelines } \\
\text { show diversity } \\
\text { regarding when to } \\
\text { start treatment (at } \\
\text { diagnosis/CD4 count } \\
\text { threshold level) [10, } \\
121-124] .\end{array}$ & $\begin{array}{l}\text { The intervention has biological } \\
\text { plausibility. ART decreases } \\
\text { the replication of human } \\
\text { immunodeficiency virus type } 1 \\
\text { and has been shown to reduce } \\
\text { the amount of HIV-1 in genital } \\
\text { secretions [37], the likely } \\
\text { mechanism for how ART reduces } \\
\text { sexual transmission of the virus } \\
\text { among MSM. } \\
\text { A consideration is the reported } \\
\text { low rates (43-84\%) of ever having } \\
\text { tested for HIV among European } \\
\text { MSM, limiting the effect of } \\
\text { serostatus-dependent prevention } \\
\text { interventions [125]. } \\
\end{array}$ & $\begin{array}{l}\text { Strong, } \\
\text { grade } 1\end{array}$ \\
\hline $\begin{array}{l}\text { Peer-led } \\
\text { group } \\
\text { interventions }\end{array}$ & UAI & $\begin{array}{l}\text { Efficacy data are consistent. A } \\
\text { systematic review including } 21 \\
\text { studies ( } n=5,197 \text { and one study } \\
\text { on unknown sample size) found a } \\
\text { significant reduction in UAl. The size } \\
\text { of the reduction ranged from } 13 \% \text { to } \\
33 \% \text { [38-40]. }\end{array}$ & \begin{tabular}{|l|} 
Available. \\
Implementation data \\
are consistent and \\
show high uptake \\
of peer-led group \\
interventions [33, 38]. \\
\end{tabular} & $\begin{array}{l}\text { Peer-led group interventions } \\
\text { for MSM have public health } \\
\text { plausibility. Acceptability and } \\
\text { uptake might be improved by the } \\
\text { involvement of peers creating } \\
\text { enabling and safe environments } \\
\text { for MSM to provide information } \\
\text { and counselling. }\end{array}$ & $\begin{array}{l}\text { Strong, } \\
\text { grade } 1\end{array}$ \\
\hline $\begin{array}{l}\text { Peer } \\
\text { outreach }\end{array}$ & UAI & $\begin{array}{l}\text { Efficacy data are consistent. A } \\
\text { systematic review including } 11 \text { studies } \\
\text { (n>7,890) reports that peer-led } \\
\text { outreach interventions are effective } \\
\text { in reducing UAl. Three meta-analysis } \\
\text { reports significant reduction in UAI } \\
\text { (OR: } 0.7 ; 95 \% \mathrm{Cl} 0.49-0.99 ; \text { OR: } 0.65 \text {; } \\
95 \% \mathrm{Cl} 0.48-0.89 \text { ), RR: } 0.70 ; 95 \% \mathrm{Cl} \\
0.55-0.91 \text { ) in comparison with no HIV } \\
\text { prevention [38]. }\end{array}$ & $\begin{array}{l}\text { Available. Peer } \\
\text { outreach is common } \\
\text { and generally well- } \\
\text { received among MSM } \\
\text { in Europe [10]. }\end{array}$ & $\begin{array}{l}\text { Peer outreach has public health } \\
\text { plausibility through that peers } \\
\text { can serve as a first point of } \\
\text { interaction to create an enabling } \\
\text { environment were persons } \\
\text { who may not seek prevention } \\
\text { interventions can be reached and } \\
\text { introduced to such interventions. }\end{array}$ & $\begin{array}{l}\text { Strong, } \\
\text { grade } 1\end{array}$ \\
\hline $\begin{array}{l}\text { Voluntary } \\
\text { testing and } \\
\text { counselling } \\
\text { for HIV }\end{array}$ & $\begin{array}{l}\text { Condom } \\
\text { use }\end{array}$ & $\begin{array}{l}\text { Efficacy data are limited. A systematic } \\
\text { review including } 11 \text { studies ( } \mathrm{n}=4,416, \\
\text { of which } 418 \mathrm{MSM} \text { ), where six studies } \\
\text { compared PLWH aware of their status } \\
\text { with PLWH unaware of their status } \\
\text { and five studies compared individuals } \\
\text { before and after seroconverting. } \\
\text { The data concluded that high-risk } \\
\text { sexual behaviour for HIV is reduced } \\
\text { after becoming aware of living with } \\
\text { HIV, reduction in UAI ranged from } \\
25 \% \text { to } 65 \% \text {. No reduction was seen } \\
\text { among those testing negative. Among } \\
\text { MSM living with HIV, studies report } \\
\text { increased condom use and decrease } \\
\text { in number of sexual partners } \\
\text { following HIV diagnosis [ } 42-45] \text {. }\end{array}$ & \begin{tabular}{|l|} 
\\
Available. \\
Acceptability for \\
testing was found to \\
be high, EMIS reports \\
that the national \\
proportion of MSM \\
reporting having had \\
an HIV-test during \\
the past 12 months \\
ranged from $20 \%$ to \\
$47 \%$, with a median of \\
$37 \%$ [35, $46-48]$.
\end{tabular} & $\begin{array}{l}\text { The intervention has biological } \\
\text { plausibility, VCT may influence } \\
\text { behaviour change through a } \\
\text { process involving acquisition } \\
\text { of HIV/AIDS knowledge and } \\
\text { learning one's HIV serostatus } \\
\text { [26]. Knowledge of HIV status } \\
\text { enables access to treatment and } \\
\text { prevention efforts dependent on } \\
\text { HIV serostatus. }\end{array}$ & $\begin{array}{l}\text { Probable, } \\
\text { grade } 2 a\end{array}$ \\
\hline
\end{tabular}

ART: antiretroviral therapy; $\mathrm{Cl}$ : confidence interval; EEA: European Economic Area; EMIS: European MSM Internet survey; EU: European Union; FTC-TDF: emtricitabine and tenofovir; LGBTI: lesbian, gay, bisexual, transgender and intersex; MSM: men who have sex with men; NA: not available; OR: odds ratio; PEP: post-exposure prophylaxis; PrEP: pre-exposure prophylaxis; PLWH: people living with HIV; RCT: randomised controlled trial; RR: relative risk; UAI: unprotected anal intercourse; US: United States; VCT: voluntary testing and counselling. 
Highest Attainable Standard of Evidence (HASTE) evaluation per HIV prevention intervention in men who have sex with men in Europe

\begin{tabular}{|c|c|c|c|c|c|}
\hline \multirow[b]{2}{*}{ Intervention } & \multirow[b]{2}{*}{ Outcome } & Efficacy data & Implementation data & Plausibility & Grading \\
\hline & & Consistent/limited/inconsistent/NA & $\begin{array}{l}\text { Available/NA from } \\
\text { European setting }\end{array}$ & $\begin{array}{l}\text { Biological plausibility / Public } \\
\text { health plausibity }\end{array}$ & $\begin{array}{l}\text { HASTE grade } \\
1-4\end{array}$ \\
\hline $\begin{array}{l}\text { Condom- } \\
\text { compatible } \\
\text { lubricant use }\end{array}$ & $\begin{array}{l}\text { Condom } \\
\text { failure }\end{array}$ & $\begin{array}{l}\text { Efficacy data are limited. Lack of } \\
\text { additional lubricants during anal } \\
\text { intercourse has been associated } \\
\text { with condom failure [49-52]. A } \\
\text { study investigating usage of } \\
915 \text { condoms at anal intercourse } \\
\text { reported a reduction in slippage } \\
\text { and breakage when using lubricants } \\
\text { [53]. Oil-based lubricants (not } \\
\text { condom-compatible) have been } \\
\text { shown to decrease tensile strength } \\
\text { and increase permeability in latex } \\
\text { condom and thereby increase } \\
\text { breakage rates [50,51]. }\end{array}$ & $\begin{array}{l}\text { Available. Studies } \\
\text { have showed that use } \\
\text { of lubricants among } \\
\text { MSM is common. } \\
\text { Distribution of condom } \\
\text { compatible lubricants } \\
\text { is feasible [54-55]. }\end{array}$ & $\begin{array}{l}\text { The intervention has biological } \\
\text { plausibility, decreasing the } \\
\text { amount of condom failure as } \\
\text { well as the amount of micro- } \\
\text { tears in rectum of the receptive } \\
\text { partner by using condom- } \\
\text { compatible lubricants at anal } \\
\text { sex might provide a protective } \\
\text { effect for HIV transmission } \\
\text { [54-56]. }\end{array}$ & $\begin{array}{l}\text { Probable, grade } \\
2 a\end{array}$ \\
\hline $\begin{array}{l}\text { Post- } \\
\text { exposure } \\
\text { prophylaxis }\end{array}$ & $\begin{array}{l}\text { HIV } \\
\text { incidence }\end{array}$ & $\begin{array}{l}\text { Efficacy data are limited. } \\
\text { Two retrospective cohort studies of } \\
\text { patients receiving PEP in Denmark } \\
(n=374) \text { and Amsterdam }(n=189) \\
\text { have been performed, each study } \\
\text { reported one seroconversion [ } 57^{-} \\
58] \text {. No adherence data was found. }\end{array}$ & $\begin{array}{l}\text { Available. } \\
\text { Implementation data } \\
\text { reports a low demand } \\
\text { for PEP in some } \\
\text { European settings, the } \\
\text { national proportion of } \\
\text { MSM who have ever } \\
\text { taken PEP ranged } \\
\text { from } 0 \% \text { to } 3.4 \% \text { with } \\
\text { a country median of } \\
1.3 \%[36,57-58] \text {. }\end{array}$ & $\begin{array}{l}\text { The intervention has a high } \\
\text { biological plausibility. ART is } \\
\text { highly effective in preventing } \\
\text { the HIV-1 virus to replicate, } \\
\text { thereby removing any virus } \\
\text { before it can establish an } \\
\text { infection. However, a low } \\
\text { demand has been noticed in } \\
\text { some European settings, which } \\
\text { might decrease the public health } \\
\text { plausibility, and information and } \\
\text { availability might need to be } \\
\text { strengthened. }\end{array}$ & $\begin{array}{l}\text { Probable, grade } \\
2 a\end{array}$ \\
\hline $\begin{array}{l}\text { Individual } \\
\text { counselling } \\
\text { for MSM } \\
\text { living with } \\
\text { HIV }\end{array}$ & UAI & $\begin{array}{l}\text { Efficacy data are limited. A cohort } \\
\text { study with one intervention }(n=146 \\
\text { MSM) and one control arm }(n=180) \\
\text { reported a significant decrease } \\
\text { in UAI among MSM with } \geq 2 \text { sex } \\
\text { partners. A study comparing } \\
\text { counselling vs standard of care } \\
\text { in a primary-care setting found } \\
\text { no difference regarding UAI at } \\
\text { six-month follow-up. A RCT of } \\
\text { peer-led individual counselling } \\
\text { intervention reported a decline in } \\
\text { HIV transmission at 6- and } 12 \text {-month } \\
\text { follow-up ( } n=249) \text { [59-61]. }\end{array}$ & $\begin{array}{l}\text { Available. } \\
\text { Implementation } \\
\text { data report that } \\
\text { acceptability and } \\
\text { uptake of individual } \\
\text { counselling is high } \\
\text { [60-61]. }\end{array}$ & $\begin{array}{l}\text { Counselling for MSM living with } \\
\text { HIV has biological plausibility, } \\
\text { through a process where } \\
\text { increased knowledge may lead } \\
\text { to behaviour change reducing } \\
\text { the risk of HIV transmission and } \\
\text { risk of acquiring STIs, which } \\
\text { might increase viral load and } \\
\text { accelerate disease progression } \\
\text { [62]. The benefit of episodic } \\
\text { or one-time intervention was } \\
\text { subject to decay over time and it } \\
\text { would need boosters to maintain } \\
\text { its effect. }\end{array}$ & $\begin{array}{l}\text { Probable, grade } \\
2 \mathrm{a}\end{array}$ \\
\hline $\begin{array}{l}\text { Individual } \\
\text { counselling } \\
\text { for MSM }\end{array}$ & UAI & $\begin{array}{l}\text { Efficacy data are inconsistent. } \\
\text { A systematic review found } \\
\text { inconsistent evidence regarding } \\
\text { the effectiveness of counselling } \\
\text { interventions in reducing UAl among } \\
\text { MSM ( }=11,636 \text { ) [38]. Two meta- } \\
\text { analysis report that HIV counselling } \\
\text { (from studies with a comparison } \\
\text { group receiving standard of care) } \\
\text { was significantly associated with } \\
\text { a reduction in UAl (OR: 0.59; } 95 \% \\
\text { Cl o.36-0.97 n=2339; OR: 0.57; } \\
95 \% \text { CI 0.37-0.87 n=4689) [39,40]. } \\
\text { Another meta-analysis found the } \\
\text { absolute effects (from studies with } \\
\text { a wait list control group) to show a } \\
\text { non-significant reduction in UAI (RR: } \\
\text { o.80; } 95 \% \text { Cl o.6o-1.06) [63]. }\end{array}$ & $\begin{array}{l}\text { Available. HIV } \\
\text { counselling } \\
\text { interventions are } \\
\text { reported to be } \\
\text { acceptable and } \\
\text { feasible among MSM } \\
\text { in Europe [36]. }\end{array}$ & $\begin{array}{l}\text { Interventions to increase } \\
\text { knowledge of HIV and } \\
\text { prevention measures have } \\
\text { public health plausibility as they } \\
\text { can influence behaviour change } \\
\text { [126]. }\end{array}$ & $\begin{array}{l}\text { Possible, grade } \\
2 b\end{array}$ \\
\hline
\end{tabular}

ART: antiretroviral therapy; Cl: confidence interval; EEA: European Economic Area; EMIS: European MSM Internet survey; EU: European Union; FTC-TDF: emtricitabine and tenofovir; LGBTI: lesbian, gay, bisexual, transgender and intersex; MSM: men who have sex with men; NA: not available; OR: odds ratio; PEP: post-exposure prophylaxis; PrEP: pre-exposure prophylaxis; PLWH: people living with HIV; RCT: randomised controlled trial; RR: relative risk; UAI: unprotected anal intercourse; US: United States; VCT: voluntary testing and counselling. 
Highest Attainable Standard of Evidence (HASTE) evaluation per HIV prevention intervention in men who have sex with men in Europe

\begin{tabular}{|c|c|c|c|c|c|}
\hline \multirow[b]{2}{*}{ Intervention } & \multirow[b]{2}{*}{ Outcome } & Efficacy data & \multirow{2}{*}{$\begin{array}{l}\text { Implementation } \\
\text { data } \\
\text { Available/NA } \\
\text { from European } \\
\text { setting }\end{array}$} & \multirow{2}{*}{$\begin{array}{l}\text { Plausibility } \\
\text { Biological plausibility / Public } \\
\text { health plausibity }\end{array}$} & \multirow{2}{*}{$\begin{array}{l}\text { Grading } \\
\text { HASTE } \\
\text { grade 1-4 }\end{array}$} \\
\hline & & Consistent/limited/inconsistent/NA & & & \\
\hline $\begin{array}{l}\text { Peer-led } \\
\text { group } \\
\text { interventions } \\
\text { targeting } \\
\text { MSM living } \\
\text { with HIV }\end{array}$ & UAI & $\begin{array}{l}\text { Efficacy data are limited. A study } \\
\text { comparing a five-session group } \\
\text { intervention focusing on promoting safe } \\
\text { sex }(n=97) \text { with a five-session standard } \\
\text { of care support group ( } n=77) \text { reports a } \\
\text { decrease in UAl among participants [64] }\end{array}$ & NA & $\begin{array}{l}\text { Public health plausibility was } \\
\text { determined. The effect of peer- } \\
\text { led group interventions may } \\
\text { improve uptake of intervention } \\
\text { and promote safe sex behaviour } \\
\text { through a combination of } \\
\text { increased knowledge, social } \\
\text { learning, influence of peers and } \\
\text { normative group behaviour [38]. }\end{array}$ & $\begin{array}{l}\text { Possible, } \\
\text { grade } 2 \mathrm{~b}\end{array}$ \\
\hline $\begin{array}{l}\text { Internet- } \\
\text { based HIV } \\
\text { prevention } \\
\text { messages }\end{array}$ & UAI & $\begin{array}{l}\text { Efficacy data are inconsistent. An RCT } \\
\text { of a persuasive computing interactive } \\
\text { intervention has shown a reduction of UAI } \\
\text { at three months but could not maintain } \\
\text { the effect at } 12 \text {-month follow-up [65]. An } \\
\text { RCT that evaluated the short-term efficacy } \\
\text { (6o days) of a low intensity digital media } \\
\text { intervention found significant reductions in } \\
\text { UAl among men exposed to videos or to a } \\
\text { website [57]. Two RCT report no differences } \\
\text { in UAI between intervention and control } \\
\text { groups [67-68]. }\end{array}$ & NA & $\begin{array}{l}\text { Interventions that increase } \\
\text { knowledge on HIV and } \\
\text { prevention measures have } \\
\text { biological plausibility. Internet } \\
\text { is one of the largest venues } \\
\text { where MSM meet sexual } \\
\text { partners [69,70]. Thereby } \\
\text { messaging on the Internet would } \\
\text { potentially reach a large number } \\
\text { of MSM. In addition, safe-sex } \\
\text { messaging on the venue where } \\
\text { MSM meet sex partners could } \\
\text { influence normative behaviour } \\
\text { around safe sex. } \\
\end{array}$ & $\begin{array}{l}\text { Possible, } \\
\text { grade } 2 b\end{array}$ \\
\hline $\begin{array}{l}\text { Interventions } \\
\text { in sex-on- } \\
\text { premises } \\
\text { venues }\end{array}$ & $\begin{array}{l}\text { UAl and } \\
\text { uptake of } \\
\text { HIV testing }\end{array}$ & $\begin{array}{l}\text { Efficacy data are limited. A study } \\
\text { performing a VCT intervention at a } \\
\text { bathhouse tested } 133 \text { men, of whom } 48 \% \\
\text { had not been tested in the previous } 12 \\
\text { months. A decrease in UAl was reported } \\
\text { three months after the intervention [73-75]. }\end{array}$ & NA & $\begin{array}{l}\text { Sex venue-based interventions } \\
\text { have public health plausibility } \\
\text { through creating easy access } \\
\text { to prevention interventions at } \\
\text { the location where men meet } \\
\text { sex partners, possibly reaching } \\
\text { MSM who do not visit service } \\
\text { sites [76,77]. Programmes may } \\
\text { create social norms that can } \\
\text { impact how MSM negotiate } \\
\text { around sexual behaviour at sex } \\
\text { venues [127,128] [47]. }\end{array}$ & $\begin{array}{l}\text { Possible, } \\
\text { grade } 2 b\end{array}$ \\
\hline $\begin{array}{l}\text { Social } \\
\text { marketing } \\
\text { interventions }\end{array}$ & $\begin{array}{l}\text { Uptake of } \\
\text { HIV-testing }\end{array}$ & $\begin{array}{l}\text { Efficacy data are limited. A systematic } \\
\text { review including three studies of cross- } \\
\text { sectional design before and after the } \\
\text { intervention reports a significant increase } \\
\text { in HIV-testing uptake (OR: } 1.58 ; 95 \% \mathrm{Cl} \\
1.40-1.77 \text { ) [78]. }\end{array}$ & NA & $\begin{array}{l}\text { Social marketing interventions } \\
\text { have public health plausibility } \\
\text { through increasing knowledge } \\
\text { on HIV and prevention measures } \\
\text { and services. Awareness } \\
\text { campaigns can also spark } \\
\text { discussions and strengthen } \\
\text { awareness, which can create a } \\
\text { change in social norms. }\end{array}$ & $\begin{array}{l}\text { Possible, } \\
\text { grade } 2 b\end{array}$ \\
\hline $\begin{array}{l}\text { Pre-exposure } \\
\text { prophylaxis }^{a}\end{array}$ & $\begin{array}{l}\text { HIV } \\
\text { incidence }\end{array}$ & $\begin{array}{l}\text { Efficacy data are limited. One multicentre } \\
\text { RCT, iPrEx, shows a } 44 \% \text { reduction in the } \\
\text { incidence of HIV }(95 \% \mathrm{Cl} 15-63 ; \mathrm{p}=0.005) \\
\text { during a } 3,324 \text { person-years follow-up } \\
\text { period among MSM. Detectable FTC-TDF } \\
\text { blood levels strongly correlated with } \\
\text { the prophylactic effect, emphasising the } \\
\text { importance of adherence to PrEP [83]. }\end{array}$ & NA & $\begin{array}{l}\text { The intervention has biological } \\
\text { plausibility. See section for } \\
\text { PEP. Little is known about } \\
\text { potential long-term side effects, } \\
\text { adherence and drug resistance. }\end{array}$ & $\begin{array}{l}\text { Possible, } \\
\text { grade } 2 b\end{array}$ \\
\hline
\end{tabular}

ART: antiretroviral therapy; $\mathrm{Cl}$ : confidence interval; EEA: European Economic Area; EMIS: European MSM Internet survey; EU: European Union; FTC-TDF: emtricitabine and tenofovir ; LGBTI: lesbian, gay, bisexual, transgender and intersex; MSM: men who have sex with men; NA: not available; OR: odds ratio; PEP: post-exposure prophylaxis; PrEP: pre-exposure prophylaxis; PLWH: people living with HIV; RCT: randomised controlled trial; RR: relative risk; UAI: unprotected anal intercourse; US: United States; VCT: voluntary testing and counselling.

\footnotetext{
${ }^{a}$ New data have been published during 2015 providing implementation data for PrEP $[115,116]$.

${ }^{\mathrm{b}}$ Assigned possible, grade $2 \mathrm{~b}$, for MSM who are only or mostly insertive during intercourse.
} 
TABLE 3D

Highest Attainable Standard of Evidence (HASTE) evaluation per HIV prevention intervention in men who have sex with men in Europe

\begin{tabular}{|c|c|c|c|c|c|}
\hline \multirow[b]{2}{*}{ Intervention } & \multirow[b]{2}{*}{ Outcome } & \multirow{2}{*}{$\begin{array}{l}\text { Efficacy data } \\
\text { Consistent/limited/ } \\
\text { inconsistent/NA }\end{array}$} & \multirow{2}{*}{$\begin{array}{l}\text { Implementation data } \\
\text { Available/NA from } \\
\text { European setting }\end{array}$} & \multirow{2}{*}{$\begin{array}{l}\text { Plausibility } \\
\text { Biological plausibility / } \\
\text { Public health plausibity }\end{array}$} & \multirow{2}{*}{$\begin{array}{l}\text { Grading } \\
\text { HASTE grade } \\
1-4\end{array}$} \\
\hline & & & & & \\
\hline $\begin{array}{l}\text { Voluntary } \\
\text { medical male } \\
\text { circumcision }^{\text {b }}\end{array}$ & $\begin{array}{l}\text { HIV } \\
\text { incidence }\end{array}$ & $\begin{array}{l}\text { Efficacy data are consistent } \\
\text { among men primarily or } \\
\text { exclusively being insertive } \\
\text { at anal sex. A Cochrane } \\
\text { comprehensive review of } 21 \\
\text { observational studies with a } \\
\text { total of } 71,693 \text { participants } \\
\text { found insufficient evidence that } \\
\text { male circumcision prevents } \\
\text { acquisition of HIV among MSM. } \\
\text { Among men having primarily or } \\
\text { exclusively insertive anal sex, } \\
\text { a significant decrease in HIV } \\
\text { infection was found (OR:0.27; } \\
95 \% \text { Cl o.17-0.44) [84]. A } \\
\text { longitudinal study reports a } \\
\text { reduced risk of HIV acquisition } \\
\text { by } 69 \% \text { among MSM who } \\
\text { reported } \geq 60 \% \text { of acts as } \\
\text { insertive with their last three } \\
\text { sex partners) [85]. }\end{array}$ & NA & $\begin{array}{l}\text { The intervention has a } \\
\text { biological plausibility } \\
\text { among MSM who are only or } \\
\text { mostly insertive during anal } \\
\text { intercourse }[85,130-133] \text {. }\end{array}$ & $\begin{array}{l}\text { Possible, grade } \\
2 b\end{array}$ \\
\hline $\begin{array}{l}\text { Training for } \\
\text { healthcare } \\
\text { providers } \\
\text { to offer } \\
\text { comprehensive } \\
\text { care for MSM }\end{array}$ & NA & NA & $\begin{array}{l}\text { NA. Many published and } \\
\text { online resources are } \\
\text { available to train health } \\
\text { providers about issues } \\
\text { facing MSM [7]. }\end{array}$ & $\begin{array}{l}\text { Training for providers to } \\
\text { offer comprehensive care } \\
\text { for MSM has public health } \\
\text { plausibility. With adequate } \\
\text { education and training, } \\
\text { healthcare providers } \\
\text { can provide appropriate } \\
\text { routine care for MSM } \\
\text { patients and help patients } \\
\text { to avoid internalising } \\
\text { stigma associated with } \\
\text { homosexuality, prevent } \\
\text { HIV acquisition, reduce } \\
\text { unsafe sex, and lead more } \\
\text { satisfying and healthy lives } \\
\text { [7]. }\end{array}$ & $\begin{array}{l}\text { Pending, grade } \\
2 \mathrm{C}\end{array}$ \\
\hline $\begin{array}{l}\text { MSM-competent } \\
\text { clinics }\end{array}$ & NA & NA & $\begin{array}{l}\text { Available. MSM clinics } \\
\text { that offer comprehensive } \\
\text { services to MSM are } \\
\text { available in many European } \\
\text { metropolitan areas and } \\
\text { have demonstrated high } \\
\text { uptake of their services } \\
\text { [86]. }\end{array}$ & $\begin{array}{l}\text { MSM-friendly clinics } \\
\text { that offer comprehensive } \\
\text { services have biological } \\
\text { plausibility through } \\
\text { removing barriers that stop } \\
\text { MSM from seeking care or } \\
\text { from disclosing relevant } \\
\text { personal information once in } \\
\text { care [87]. }\end{array}$ & $\begin{array}{l}\text { Pending, grade } \\
2 C\end{array}$ \\
\hline $\begin{array}{l}\text { Voluntary } \\
\text { anonymous } \\
\text { partner } \\
\text { notification }\end{array}$ & $\begin{array}{l}\text { HIV } \\
\text { incidence }\end{array}$ & NA & $\begin{array}{l}\text { Available. Acceptability, } \\
\text { defined as willingness of } \\
\text { index patients to notify } \\
\text { their sex partners, has } \\
\text { been shown to be high } \\
\text { among MSM in Europe } \\
{[88,89] \text {. }}\end{array}$ & $\begin{array}{l}\text { Contract tracing has } \\
\text { biological plausibility by } \\
\text { enabling early diagnosis, } \\
\text { treatment and care, } \\
\text { which benefits the } \\
\text { individual person as well } \\
\text { as likely interrupting the } \\
\text { transmission chain, thereby } \\
\text { reducing incidence. }\end{array}$ & $\begin{array}{l}\text { Pending, grade } \\
2 C\end{array}$ \\
\hline $\begin{array}{l}\text { Campaigns } \\
\text { for lesbian, } \\
\text { gay, bisexual, } \\
\text { transgender } \\
\text { and intersex } \\
\text { equality }\end{array}$ & $\begin{array}{l}\text { Self- } \\
\text { reported } \\
\text { stigma } \\
\text { towards } \\
\text { LGBTI }\end{array}$ & $\begin{array}{l}\text { No studies have fully evaluated } \\
\text { structural interventions for } \\
\text { MSM. Education programmes } \\
\text { focusing on changing straight- } \\
\text { identified persons' perceptions } \\
\text { and challenging gender } \\
\text { norms have been shown to be } \\
\text { successful in decreasing stigma } \\
\text { [90,91]. }\end{array}$ & NA & $\begin{array}{l}\text { Anti-stigma and LGBTI rights } \\
\text { promotion has public health } \\
\text { plausibility by removing } \\
\text { structural barriers and } \\
\text { providing a climate where } \\
\text { MSM can access preventive } \\
\text { service without fear of } \\
\text { stigma [134]. }\end{array}$ & $\begin{array}{l}\text { Pending, grade } \\
\text { 2C }\end{array}$ \\
\hline
\end{tabular}

ART: antiretroviral therapy; CI: confidence interval; EEA: European Economic Area; EMIS: European MSM Internet survey; EU: European Union; FTC-TDF: emtricitabine and tenofovir ; LGBTI: lesbian, gay, bisexual, transgender and intersex; MSM: men who have sex with men; NA: not available; OR: odds ratio; PEP: post-exposure prophylaxis; PrEP: pre-exposure prophylaxis; PLWH: people living with HIV; RCT: randomised controlled trial; RR: relative risk; UAI: unprotected anal intercourse; US: United States; VCT: voluntary testing and counselling.

${ }^{a}$ New data have been published during 2015 providing implementation data for PrEP [115, 116].

${ }^{b}$ Assigned possible, grade $2 b$, for MSM who are only or mostly insertive during intercourse. 
Highest Attainable Standard of Evidence (HASTE) evaluation per HIV prevention intervention in men who have sex with men in Europe

\begin{tabular}{|c|c|c|c|c|c|}
\hline \multirow[b]{2}{*}{ Intervention } & \multirow[b]{2}{*}{ Outcome } & Efficacy data & $\begin{array}{l}\text { Implementation } \\
\text { data }\end{array}$ & Plausibility & Grading \\
\hline & & $\begin{array}{l}\text { Consistent/limited/ } \\
\text { inconsistent/NA }\end{array}$ & $\begin{array}{l}\text { Available/NA } \\
\text { from European } \\
\text { setting }\end{array}$ & $\begin{array}{l}\text { Biological plausibility / Public health } \\
\text { plausibity }\end{array}$ & $\begin{array}{l}\text { HASTE grade } \\
1-4\end{array}$ \\
\hline $\begin{array}{l}\text { Female } \\
\text { condom use } \\
\text { at anal sex }\end{array}$ & $\begin{array}{l}\text { Condom } \\
\text { failure }\end{array}$ & $\begin{array}{l}\text { Further evidence is needed to } \\
\text { establish efficacy. } \\
\text { One study reports no } \\
\text { significant difference regarding } \\
\text { condom breakage at anal sex } \\
\text { compared with male condoms, } \\
\text { but a higher rate of condom } \\
\text { slippage OR: } 2.7 ; 95 \% \mathrm{Cl} \\
1.2-5.8)(\mathrm{n}=76) \text { in comparison } \\
\text { to male condoms [92]. }\end{array}$ & NA & $\begin{array}{l}\text { The intervention has biological plausibility as } \\
\text { a barrier method. Female condoms potentially } \\
\text { offer a protection method controlled by the } \\
\text { receptive partner at anal sex. Higher rates of } \\
\text { slippage, pain and discomfort when using the } \\
\text { female condom at anal sex has been reported } \\
\text { compared with using male latex condoms } \\
\text { which is a potential risk/harm with female } \\
\text { condom use at anal sex [92]. There is a need } \\
\text { for safety and efficacy studies of a female } \\
\text { condom designed for anal sex. }\end{array}$ & $\begin{array}{l}\text { Pending, } \\
\text { grade 2C }\end{array}$ \\
\hline Serosorting & $\begin{array}{l}\text { HIV } \\
\text { incidence }\end{array}$ & $\begin{array}{l}\text { Efficacy data are inconsistent. } \\
\text { A systematic review included } \\
\text { three observational studies } \\
\text { found that serosorting } \\
\text { increased HIV transmission } \\
\text { by } 79 \% \text { compared with } \\
\text { condom use [27]. However, } \\
\text { compared with no condom } \\
\text { use serosorting reduced HIV } \\
\text { transmission by } 53 \% \text { [27,93]. } \\
\text { The Explore trial performed } \\
\text { among MSM in six US cities } \\
\text { found that serosorting was } \\
\text { associated with a modest } \\
\text { reduction in HIV acquisition for } \\
\text { HIV-negative MSM (OR: 0.88; } \\
95 \% \text { Cl o.81-0.95) [94]. }\end{array}$ & $\begin{array}{l}\text { NA. European } \\
\text { men have } \\
\text { reported in } \\
\text { studies that they } \\
\text { use serosorting } \\
\text { as a risk } \\
\text { management } \\
\text { approach }[95,96] \text {. }\end{array}$ & $\begin{array}{l}\text { Serosorting may not have an effect due to } \\
\text { low testing rates and the low possibility of } \\
\text { detecting primary HIV infection. Public health } \\
\text { plausibility is undefined, There is a risk that } \\
\text { individuals may rely on a negative HIV-testing } \\
\text { result that is not accurate. }\end{array}$ & $\begin{array}{l}\text { Insufficient, } \\
\text { grade } 3\end{array}$ \\
\hline $\begin{array}{l}\text { Avoiding } \\
\text { semen in } \\
\text { the mouth/ } \\
\text { unprotected } \\
\text { oral sex }\end{array}$ & $\begin{array}{l}\text { HIV } \\
\text { incidence }\end{array}$ & $\begin{array}{l}\text { Efficacy data are inconsistent. } \\
\text { One prospective cohort study } \\
\text { including } 2,189 \text { high risk MSM } \\
\text { in the US between } 1992 \text { and } \\
1994 \text { (2,633 person-years) } \\
\text { reports a 0.06\% risk of HIV at } \\
\text { receptive oral sex with a sexual } \\
\text { partner living with HIV and } \\
\text { a o.04\% ( } 95 \% \text { Cl o.01-0.17) } \\
\text { risk with a sexual partner. A } \\
\text { cross-sectional study including } \\
239 \text { MSM reporting only having } \\
\text { oral sex over the past six } \\
\text { months (1999-2001) detected } \\
\text { no cases of HIV. Observational } \\
\text { studies from several high- } \\
\text { income country settings have } \\
\text { reported cases of self-reported } \\
\text { oral transmission of HIV [102, } \\
135-137] \text {. }\end{array}$ & NA & $\begin{array}{l}\text { There is biological plausibility that not taking } \\
\text { semen in the mouth and thereby limiting } \\
\text { the contact between semen with possible } \\
\text { HIV virus content and the oral mucosa could } \\
\text { potentially remove this opportunity for } \\
\text { transmission. However, transmission rates } \\
\text { reported are between o\% and } 0.04 \% \text {, which } \\
\text { is lower than estimated per contact risk } \\
\text { of HIV at receptive anal intercourse with a } \\
\text { condom (o.18\%; } 95 \% \text { Cl o.10-0.28\%). The } \\
\text { low risk of HIV transmission implies that } \\
\text { the avoidance of taking semen in the mouth } \\
\text { would not have any significant effect. }\end{array}$ & $\begin{array}{l}\text { Insufficient, } \\
\text { grade } 3\end{array}$ \\
\hline
\end{tabular}

ART: antiretroviral therapy; $\mathrm{Cl}$ : confidence interval; EEA: European Economic Area; EMIS: European MSM Internet survey; EU: European Union; FTC-TDF: emtricitabine and tenofovir; LGBTI: lesbian, gay, bisexual, transgender and intersex; MSM: men who have sex with men; NA: not available; OR: odds ratio; PEP: post-exposure prophylaxis; PrEP: pre-exposure prophylaxis; PLWH: people living with HIV; RCT: randomised controlled trial; RR: relative risk; UAI: unprotected anal intercourse; US: United States; VCT: voluntary testing and counselling. 
Highest Attainable Standard of Evidence (HASTE) evaluation per HIV prevention intervention in men who have sex with men in Europe

\begin{tabular}{|c|c|c|c|c|c|}
\hline \multirow[b]{2}{*}{ Intervention } & \multirow[b]{2}{*}{ Outcome } & Efficacy data & $\begin{array}{l}\text { Implementation } \\
\text { data }\end{array}$ & Plausibility & Grading \\
\hline & & $\begin{array}{l}\text { Consistent/limited/ } \\
\text { inconsistent/NA }\end{array}$ & $\begin{array}{l}\text { Available/NA } \\
\text { from European } \\
\text { setting }\end{array}$ & Biological plausibility /Public health plausibity & $\begin{array}{l}\text { HASTE } \\
\text { grade } 1-4\end{array}$ \\
\hline $\begin{array}{l}\text { Avoiding nitrite } \\
\text { inhalants/poppers } \\
\text { at UAl }\end{array}$ & NA & NA & $\begin{array}{l}\text { NA. Consistent } \\
\text { high usage of } \\
\text { poppers across } \\
\text { European } \\
\text { settings was self- } \\
\text { reported in the } \\
\text { EMIS } 2010 \text { study } \\
\text { [35]. }\end{array}$ & $\begin{array}{l}\text { The pathway by which nitrite inhalants could } \\
\text { lead to transmission of HIV is unclear. Nitrites } \\
\text { inhalants cause peripheral vasodilatation and } \\
\text { are believed to decrease anal sphincter tone, } \\
\text { potentially leading to more traumatic sexual } \\
\text { intercourse or more direct exposure to HIV target } \\
\text { cells [98]. There are limited animal and human } \\
\text { data suggesting that nitrite inhalants may cause } \\
\text { transient immunosuppression or alter cytokine } \\
\text { profiles, which could enhance transmission of } \\
\text { HIV across mucosal barriers [99, 100]. Nitrite } \\
\text { inhalants have been reported to be associated } \\
\text { with high-risk sexual behaviour for STI/HIV } \\
\text { including higher number of partners [101-103]. } \\
\text { Frequent use of sex drugs may imply a high- } \\
\text { risk marker of behavioural disinhibition that } \\
\text { includes receptive UAl with multiple partners } \\
\text { [98,104]. Limited evidence supporting biological } \\
\text { plausibility was found. }\end{array}$ & $\begin{array}{l}\text { Insufficient, } \\
\text { grade } 3\end{array}$ \\
\hline $\begin{array}{l}\text { Interventions to } \\
\text { reduce alcohol } \\
\text { binge drinking }\end{array}$ & UAI & $\begin{array}{l}\text { Efficacy data are } \\
\text { inconsistent. A RCT } \\
\text { study evaluated a } \\
\text { combined intervention } \\
\text { among MSM living } \\
\text { with HIV promoting } \\
\text { two target behaviours, } \\
\text { abstinence from/ } \\
\text { reduction in alcohol } \\
\text { use and safe sex } \\
\text { practices, compared } \\
\text { with an unexposed } \\
\text { control group reports } \\
\text { no effect on UAl } \\
\text { (n=253) [105]. }\end{array}$ & NA & $\begin{array}{l}\text { There is plausibility that alcohol binge drinking } \\
\text { through disinhibition can lead to increased } \\
\text { sexual risk behaviour [138]. Thus, behavioural } \\
\text { interventions that decrease alcohol consumption } \\
\text { might lead to decrease in UAl. }\end{array}$ & $\begin{array}{l}\text { Insufficient, } \\
\text { grade } 3\end{array}$ \\
\hline
\end{tabular}

ART: antiretroviral therapy; CI: confidence interval; EEA: European Economic Area; EMIS: European MSM Internet survey; EU: European Union; FTC-TDF: emtricitabine and tenofovir ; LGBTI: lesbian, gay, bisexual, transgender and intersex; MSM: men who have sex with men; NA: not available; OR: odds ratio; PEP: post-exposure prophylaxis; PrEP: pre-exposure prophylaxis; PLWH: people living with HIV; RCT: randomised controlled trial; RR: relative risk; UAI: unprotected anal intercourse; US: United States; VCT: voluntary testing and counselling.

information and peer support, are associated with a $30 \%$ reduction in UAl compared with minimal or no HIV prevention [38]. Peer outreach is common and generally well-received among MSM in Europe [10]. The intervention was judged plausible as peers can serve as a first point of interaction to create an enabling environment were persons who may not seek prevention interventions can be reached and introduced to interventions such as counselling, HIV/STI testing and treatment.

\section{Interventions assigned a probable}

\section{recommendation (HASTE grade 2a)}

HASTE grade $2 a$ was assigned to four interventions: Voluntary counselling and testing for HIV, condomcompatible lubricant, post-exposure prophylaxis (PEP), and individual counselling for MSM living with HIV.

\section{Voluntary testing and counselling for HIV}

A systematic review performed in 2005 including 11 studies $(n=4,416$, of which 418 MSM), where six studies compared PLWH aware of their status with PLWH unaware of their status, and five studies compared individuals before and after seroconverting. The data concluded that high-risk sexual behaviour for HIV is reduced after becoming aware of living with HIV, reduction in UAI ranged from $25 \%$ to $65 \%$, but no reduction was seen among those testing negative. Among MSM living with HIV, studies report increased condom use and decrease in number of sexual partners following HIV diagnosis and counselling [42-45].

Acceptability for testing was found to be high, EMIS reports that the national proportion of MSM reporting having had an HIV-test during the past 12 months ranged from $20 \%$ to $47 \%$, with a country median of 
$37 \%$. However, the proportion that were 'quite' or 'very' confident they could access an HIV test if they wanted one ranged from $73 \%$ to $96 \%$ with a median of $91 \%$ [35,46-48]. Plausibility was determined as VCT may influence behaviour through a process involving acquisition of HIV/AIDS knowledge and learning one's HIV serostatus [45]. In addition, knowledge of HIV status enables access to ART and care, reducing onward transmission.

\section{Condom-compatible lubricant use}

Lack of additional lubricants during anal intercourse has been associated with condom failure [49-52]. A study investigating usage of 915 condoms at anal intercourse reported a reduction in slippage and breakage when using lubricants [53]. However, oil-based lubricants (not condom-compatible) have been shown to decrease tensile strength and increase permeability in latex condom and increase breakage rates $[50,51]$. Implementation data reports that lubricant use among MSM is high [36]. The intervention was judged plausible by the decrease in condom failure as well as that the amount of micro-tears in rectum of the receptive partner may be diminished by using condom-compatible lubricants [54-56].

Post-exposure prophylaxis

PEP, defined as the administration of ART starting within 72 hours post exposure and prolonged for 28 days, was evaluated by two retrospective cohort studies of patients receiving PEP in Denmark $(n=374)$ and Amsterdam $(n=189)$, each study reported one seroconversion [57,58]. No adherence data were found. Implementation data reports a low demand for PEP in some European settings, although it is considered the standard of care. The national proportion of MSM who have ever taken PEP ranged from $0 \%$ to $3.4 \%$ with a country median of $1.3 \%$ [36]. Low demand and uptake limit the public health impact of PEP. Information about and availability of PEP might need to be strengthened $[57,58]$. Plausibility is determined by the effect of ART post-exposure (within 72 hours) that diminishes the HIV-virus before an infection can be established.

Individual counselling for men who have sex with men living with HIV

A cohort study comparing an intervention group receiving individual counselling on risk reduction for HIV by a trained counsellor $(n=146)$ and one control group $(n=180)$ reported a significant decrease in UAI among MSM living with HIV with more than two sexual partners. Another RCT of peer-led individual counselling intervention reported a decline in sexual risk behaviour for HIV at 6 and 12 months follow-up $(n=249)$ [59-61]. Implementation data report that acceptability and uptake of individual counselling are high $[60,61]$. Plausibility is determined through a process where increased knowledge may lead to behaviour change reducing the risk of HIV transmission and risk of acquiring STIs that might increase viral load and accelerate disease progression [62].
Interventions assigned a possible recommendation (HASTE grade $2 \mathbf{b}$ )

An additional seven interventions were graded HASTE grade $2 \mathrm{~b}$ including: individual counselling for MSM, peer-led group interventions targeting MSM living with HIV, Internet-based HIV prevention messages, interventions in sex-on-premises venues, social marketing interventions, pre-exposure prophylaxis (PrEP) and voluntary medical male circumcision.

Individual counselling for men who have sex with men Two meta-analyses examining individual counselling on HIV risk reduction with a comparison group receiving standard care found significant $41 \%$ and $43 \%$ reductions in UAI (OR: 0.59; $95 \% \mathrm{Cl} 0.36-0.97, n=2,339$; OR: $0.57 ; 95 \% \mathrm{Cl} 0.37-0.87, \mathrm{n}=4,689)[39,40]$. A recent systematic review which included both these studies reports inconsistent evidence regarding the effectiveness of individual counselling in reducing UAI among MSM $(n=11,636)$ [38]. This is due to the inclusion of another meta-analysis that found individual counselling clients (compared with waiting list control group) to report a non-significant $20 \%$ reduction in UAI (RR: $0.80 ; 95 \% \mathrm{Cl} 0.60-1.06)$ [63]. HIV counselling interventions are reported to be acceptable and feasible among MSM in Europe [36]. Plausibility is defined as interventions that increase knowledge of HIV and prevention measures can influence behaviour change.

Peer-led group interventions targeting men who have sex with men living with HIV

Peer-led group interventions among MSM living with HIV are defined as interactive group activities facilitated by a trained peer. A study comparing a five-session group intervention focusing on promoting safe sex $(n=97)$ with a five-session standard of care support group $(n=77)$ reports a decrease in UAI among participants [64]. Implementation data were not available. Plausibility was determined as the effect of peer-led group interventions may improve uptake by creating enabling and safe environments for MSM and promote safe sex behaviour through a combination of increased knowledge, social learning, influence of peers and normative group behaviour [38].

Internet-based HIV prevention messages

The Internet is a special venue in the sense that apart from being another potential meeting-dating venue, it may also be a venue for private and anonymous access to sexual health and well-being, at your own choice of time and physical place. Since specific longitudinal online interventions exist that are exclusively for use on the Internet, it was deemed important to review Internet-based interventions separately from other sex-venue based interventions.

An RCT of a persuasive computing interactive HIV messaging intervention has shown a reduction of UAI at three-month follow-up, but could not maintain the effect at 12-month follow-up [65]. An RCT that evaluated the short-term efficacy (6o days) of a low-intensity 
digital media HIV messaging intervention found significant reductions in UAI among men exposed to videos or to a website [66]. Two RCTs report no differences in UAI between an intervention group receiving Internet-based messaging and non-exposed control groups $[67,68]$. The Internet is a common setting for MSM to meet sexual partners [69-72]. Messaging on the Internet would potentially reach a large number of MSM. The intervention was judged plausible as safe sex messaging on the online venue where MSM meet sex partners could influence normative behaviour around safer sex practices.

\section{Interventions in sex-on-premises venues}

Interventions in sex-on-premises venues are defined as prevention activities such as information, counselling and VCT at venues where MSM gather and seek sexual partners. A VCT intervention at a bathhouse tested 133 men of whom $48 \%$ had not been tested in the previous 12 months. A decrease in UAI was reported 3 months after the intervention, which highlights the prevention effect of HIV testing among those never tested before [73-75]. Implementation data were not available. Sex venue-based interventions have public health plausibility through creating easy access to prevention interventions at a location where MSM gather and meet sex partners, possibly reaching MSM who do not visit service sites [76,77].

\section{Social marketing interventions}

A systematic review of social marketing strategies promoting HIV testing (including three intervention evaluations of cross-sectional before-and-after design) reports a significant increase in HIV-testing uptake (OR: $1.58 ; 95 \% \mathrm{Cl}$ 1.40-1.77) [78]. Social marketing interventions include media messaging (any form of media) grounded in social marketing theory [79-82]. Implementation data were not available. Public health plausibility is achieved through increasing knowledge of HIV and prevention measures and services, through manipulation of perceptions of the desirability of precautions (and the undesirability of risks) and through the promotion of social norms for precaution.

\section{Pre-exposure prophylaxis}

One multicentre RCT, iPrEx, evaluated the efficacy of once-daily oral emtricitabine and tenofovir (FTC-TDF, Truvada) among men and transgender women who have sex with men $(n=1,251)$ compared with placebo $(n=1,224)$ for the prevention of HIV acquisition. One hundred people became infected during the follow-up period of 1.8 years (median, 1.2 years; maximum, 2.8 years, total of 3,324 person-years). Thirty six in the FTC-TDF group and 64 in the placebo group became infected, which indicates a $44 \%$ reduction in the incidence of HIV ( $95 \% \mathrm{Cl} 15-63 ; p=0.005)$. In the FTC-TDF group, the study drug was detected in 22 of 43 of seronegative subjects (51\%) and in 3 of $34 \mathrm{HIV}$-infected subjects (9\%) (p<0.001) [83]. Detectable FTC-TDF blood levels strongly correlated with the prophylactic effect, emphasising the importance of adherence to
PreP. Little is known about potential long-term side effects, adherence, impact on other risk behaviour and drug resistance. Biological plausibility is determined; ART is highly effective in preventing replication of the HIV-1 virus, and so its presence could remove any virus before an infection can be established.

Voluntary medical male circumcision

A Cochrane comprehensive review of 21 observational studies with a total of 71,693 participants found insufficient evidence that male circumcision prevents acquisition of HIV among MSM [84]. However, among men having primarily or exclusively insertive anal sex, there was a significant decrease in HIV infection (OR: 0.27; $95 \% \mathrm{Cl} 0.17-0.44$ ) [84]. The longitudinal study suggested that it reduced risk of HIV acquisition by $69 \%$ among MSM who reported $\geq 60 \%$ of acts as insertive with their last three partners [85]. Programmatic issues such as safety of male circumcision, sexual behaviour following male circumcision, and sexual satisfaction and function have not been addressed specifically among MSM. No implementation data were found. The effect is plausible among MSM who are only or mostly insertive during anal intercourse, which would comprise a limited group of MSM. Therefore, the intervention receives a grade of $2 b$ for MSM who are only or mostly insertive during anal intercourse.

Interventions assigned a pending recommendation (HASTE grade 2c)

A HASTE grade $2 \mathrm{C}$ was assigned to five interventions: training for healthcare providers to offer comprehensive care for MSM, MSM-competent health clinics, voluntary anonymous partner notification, campaigns for lesbian, gay, bisexual, transgender and intersex equality (LGBTI), and female condom use for anal intercourse.

Training for healthcare providers to offer comprehensive care for men who have sex with men Published and online resources are available to train health providers about issues facing MSM, but no evaluation study was found [7]. Implementation data were not available. Plausibility was deemed strong because training providers can offer comprehensive care for MSM, including appropriate routine care for MSM patients, and help patients to avoid internalising stigma associated with homosexuality, prevent HIV and other STI acquisition, and lead more satisfying and healthy lives [7].

MSM-competent health clinics

MSM-competent health clinics that offer comprehensive services to MSM are available in many European metropolitan areas and have demonstrated high uptake of their services [86]. MSM-competent health clinics that offer comprehensive services have plausibility through removing barriers that prevent MSM from seeking care or from disclosing relevant personal information once in care [87]. 
Voluntary anonymous partner notification

No studies evaluating voluntary anonymous partner notification were found. Acceptability, defined as willingness of index patients to notify their sex partners about living with HIV, has been shown to be high among MSM in Europe $[88,89]$. Voluntary anonymous partner notification has a plausible effect by enabling early diagnosis, treatment and care, which benefits the individual person as well likely interrupting the transmission chain, thereby reducing HIV incidence.

Campaigns for lesbian, gay, bisexual, transgender and intersex equality

No studies have fully evaluated structural interventions for MSM defined as activities promoting equality through education, media awareness campaigns and policy regarding an HIV-preventative effect. Education programmes focusing on changing straight-identified men and women's perceptions of the heterosexual majority and challenging gender norms have been shown to be successful in decreasing stigma against LGBTI $[90,91]$. Implementation data are not available. Anti-stigma and LGBTI rights promotion have public health plausibility by removing structural barriers and providing a climate where MSM can access preventive and care service without fear of stigma.

\section{Female condom use for anal intercourse}

In comparisons with male condoms, one study reports the female condom to have no significantly different breakage at anal intercourse, but to have a higher rate of slippage OR: $2.7 ; 95 \% \mathrm{Cl} 1.2-5.8(n=76)$ [92] No implementation data from Europe were found. The intervention has plausibility as a barrier method for HIV transmission. Female condoms potentially offer a protection method controlled by the receptive partner at anal intercourse. Higher rates of slippage, pain and discomfort when using the female condom at anal intercourse have been reported compared with using male latex condoms, which is a potential risk/harm with female condom use at anal intercourse [92]. There is a need for safety and efficacy studies of a female condom developed particularly for anal intercourse.

\section{Interventions assigned an insufficient} recommendation (HASTE grade 3)

An insufficient level of evidence available, HASTE grade 3, was assigned to four interventions: serosorting, avoiding taking semen in the mouth/unprotected oral sex, avoiding use of poppers at UAI and avoiding alcohol binge drinking.

Serosorting

A systematic review including three observational studies [27] found that serosorting (i.e. only engaging in unprotected intercourse with individuals thought to have the same HIV status), increased HIV transmission by $79 \%$ compared with condom use. However, compared with no condom use, serosorting reduced HIV transmission by $53 \%[27,93]$. The Explore trial performed among MSM in six cities in the United States
(US) found that serosorting was associated with a modest reduction in HIV acquisition for HIV-negative MSM (OR: 0.88; 95\% Cl 0.81-0.95) [94]. Some European men have reported in studies that they use serosorting as a risk management approach [95, 96]. Serosorting may not have an effect due to low testing rates and the low possibility of detecting primary HIV infection. There is a risk that individuals may rely on a negative HIV test result that is not accurate. Serosorting among people living with HIV can be associated with an increased risk of STIs, which have been shown to cause a peak in HIV viral load in semen among individuals on ART, which could affect HIV onward transmission [97]. Public health plausibility is undefined.

Avoiding semen in the mouth/unprotected oral sex A prospective cohort study including 2,189 high-risk MSM in the US between 1992 and 1994 (2,633 person years) reported a $0.06 \%$ risk of HIV at receptive oral sex with a sexual partner living with HIV and a $0.04 \%$ (95\% $\mathrm{Cl}$ 0.01-0.17) risk with a sexual partner of unknown serostatus [102]. A cross-sectional study including 239 MSM reporting only oral sex over the past six months (1999-2001) detected no HIV. No implementation data are available [136].

There is biological plausibility that not taking semen in the mouth and thereby limiting the contact between semen with possible HIV virus content and the oral mucosa could potentially remove this opportunity for transmission. However, transmission rates reported are between $0 \%$ and $0.04 \%$, which is lower than estimated per contact risk of HIV at receptive anal intercourse with a condom (0.18\%; $95 \% \mathrm{Cl} 0.10-0.28)$ The low risk of transmission implies that the avoidance of taking semen in the mouth would not have a significant effect on transmission [11].

Avoiding use of nitrite inhalants/poppers at unprotected anal intercourse

No efficacy data were available. Consistent high usage of poppers across European settings was self-reported in the EMIS 2010 study [36]. The pathway by which nitrite inhalants could lead to transmission of HIV transmission is unclear. Nitrite inhalants cause peripheral vasodilatation and are believed to decrease anal sphincter tone, potentially leading to more traumatic sexual intercourse or more direct exposure to HIV target cells [98]. There are limited animal and human data suggesting that nitrite inhalants may cause transient immunosuppression or alter cytokine profiles, which could enhance transmission of HIV across mucosal barriers $[99,100]$. Nitrite inhalants have been reported to be associated with high-risk sexual behaviour for STI/ HIV including higher number of partners [101-103]. Frequent use of sex drugs may imply a high-risk marker of behavioural disinhibition that includes unprotected receptive anal intercourse with multiple partners $[98,104]$. Thereby, there is limited evidence supporting biological plausibility. 
Interventions to reduce alcohol binge drinking

An RCT evaluated a combined intervention among MSM living with HIV promoting two target behaviours, abstinence from/reduction in alcohol use and safe sex practices, compared with an unexposed control group $(n=253)$. The intervention had no effect on UAI [105]. Implementation data were not available. There is plausibility that alcohol binge drinking may cause disinhibition that can lead to increased sexual risk behaviour. Thus, behavioural interventions that decrease alcohol consumption might lead to a decrease in UAI.

\section{Discussion}

This systematic review of HIV prevention interventions among MSM found that four of the 24 interventions reviewed could be assigned a HASTE grade 1, equal to a strong recommendation. Another four interventions could be assigned grade $2 a$, equal to a probable recommendation. In addition, another seven interventions were assigned grade $2 \mathrm{~b}$, a possible recommendation. Unambiguous recommendations can be made to MSM to use condoms and condom-compatible lubricant when engaging in anal intercourse, to test frequently for HIV and STIs, to use ART if living with HIV, and, if uninfected, to use PEP if exposed to HIV. Recommendations can be made to service commissioners and providers to provide MSM with access to HIV testing, to provide medical care including ART to PLWH, to provide PEP to those not infected, and to provide or make otherwise accessible condoms and lubricant. Interventions which promote HIV testing, condom use, ART and PEP can also be recommended. Evidence-based delivery modes include peer-led interventions, educational outreach and group work programmes, with specific peer-led programmes for men living with HIV.

An important consideration in HIV prevention programme planning is that there are synergies and dependency between the recommended interventions, indicating that combining interventions into programmes is desirable [106]. For example, biomedical interventions dependent on HIV serostatus (e.g. ART, PrEP, PEP) need to be implemented in combination with easy access to the provision of VCT. An HIV-testing service itself can achieve high coverage through peer outreach and social marketing. Therefore, interventions should be packaged together to enhance their potential full effect to prevent HIV.

There was a striking lack of European effectiveness studies, where interventions are examined outside an RCT setting. Additional research into the areas of effectiveness in the European context is needed inform HIV prevention decision-making and programme planning. These are required both regarding new interventions, such as the implementation of PrEP programmes, and to report results of follow-up on already-implemented interventions such as early initiation of ART, PEP, and voluntary anonymous partner notification.
The challenge of scaling up ART for MSM with HIV in Europe includes both more widespread and more frequent HIV-testing, as well as increasing ART accessibility to men testing positive. In $2013,37 \%$ of the MSM diagnosed with HIV in the EU/EEA were diagnosed late (defined as $\mathrm{CD}_{4}$ cell count $<350 / \mu \mathrm{L}$ ), indicating that many men who acquire HIV are unaware of their infection for some time [107]. Models using data from the 2010 United Kingdom national cohort of MSM living with HIV suggest that extending ART to MSM diagnosed with HIV with $\mathrm{CD}_{4}$ counts $\measuredangle 500$ cells $/ \mu \mathrm{L}$ would have reduced the overall proportion of infectious men from $35 \%$ to $29 \%$. However reducing the undiagnosed population by $50 \%$ would have reduced this to $21 \%$, which serves to emphasise the importance of frequent HIV testing [108].

Comprehensive community education programmes linking peer community outreach work with easy access to HIV-testing and treatment are key components of universal coverage of antiretroviral treatment and treatment as prevention. As HIV self-tests become authorised for use in European countries, they may contribute to increased testing and linkage to care. A French study reported that accessing an unauthorised HIV self-test was associated with living one's sex life with men in total secrecy and having had unprotected anal intercourse with men during the last 12 months, indicating that for particular groups of MSM, autonomous self-testing may reduce barriers to testing [109]. The majority of literature on VCT included in this review was published before 2000 , indicating a need for more contemporary published studies evaluating delivery of HIV testing among MSM in Europe.

Drug approval by the European Medicines Agency for emtricitabine and tenofovir disoproxil fumarate (TDF/ FTC), brand-named Truvada, to be used for pre-exposure prophylaxis is currently pending. It has been approved by the US Food and Drug Administration since July 2012. Studies among MSM in France and the UK have showed a high interest in and acceptability for PreP among MSM [110,111]. Half of 842 HIV-negative MSM in London reported that they would consider using PrEP if it became available as a daily pill [111]. The longterm health effects of TDF/FTC in HIV-uninfected men and men who become HIV-infected while taking PrEP needs evaluation [112-114]. The PROUD clinical trial in the UK and the IPERGAY clinical trial in France and Canada report that PrEP is highly protective against HIV acquisition among HIV-negative MSM and that PrEP use was not associated with increased number of sexual partners, decreased condom use, or increased incidence of STIs $[115,116]$. PROUD and IPERGAY data were not available when this review was performed but should be taken into account when providing guidance on PrEP.

In Europe, structural barriers including human rights violations, homophobia, direct and indirect discrimination and obstructive policies and laws all limit the 
effectiveness of HIV intervention programmes, by reducing service uptake and by compromising the quality of services. In the European Survey of Lesbian, Gay, Bisexual and Transgender persons conducted in 2012, $38 \%$ of European MSM respondents said that they were not open with any healthcare provider about their sexual orientation; the percentage of men saying this was $70 \%$ or higher in several EU countries, including Lithuania, Slovakia, Romania, Poland, and Latvia [117]. Structural interventions aiming to decrease stigma and discrimination against MSM could result in an open climate where MSM feel safe to disclose their sexual practice and enrol in prevention and treatment programmes. Evaluation research is needed to guide how structural interventions for MSM in Europe would best be designed and implemented. As LGBTI rights improve in diverse European settings there will be opportunities for evaluating the health impacts that might be achieved due to structural and policy changes, and these should not be missed.

More descriptive data are needed on morbidity, wellbeing and health service use among MSM and MSM sub-populations. To minimise selection bias inherent in sampling strategies such as purposive or voluntary recruitment, combinations of sampling strategies that complement each other may increase validity. These may include the inclusion of sexual identity and practice variables in service monitoring, respondent driven sampling in real-life or on the web and time-location sampling.

This systematic review of HIV prevention interventions among MSM aimed for a comprehensive evidencebased multidisciplinary approach. The HASTE grading framework that is designed to evaluate HIV interventions among MSM allowed for an inclusive approach employing three tiers of data, and was particularly helpful for highlighting the importance of implementation data. In the grading process, we spent time thoroughly discussing the differences between HASTE grade $2 a$ probable, $2 b$ possible and $2 c$ pending for recommendation. These grades overlap somewhat, and careful consideration is required when assigning them.

Behavioural and biological outcomes were assigned the same value according to the inclusion criteria for this review. As HIV incidence studies are rare, this review argues that all available efficacy data are relevant to include if the specific outcome variables are transparently reported. Reliance on self-reports of sexual risk behaviour is however subject to recall bias and social desirability bias, which may have diluted the measured effects of some interventions [118]. Most studies applied a short recall duration, which has been shown to maximise self-report accuracy and thereby diminish recall bias [119].

In this review it was notable that studies usually evaluated a mix of different (often related or entangled) interventions rather than a single component intervention. Similarly, outcome data in intervention studies are usually combined without disaggregating results by, for example, knowledge of partners' HIV status. Hence the effects of different individual components as well as effects in MSM sub-groups might be diluted in some results.

Serosorting was assigned an insufficient grade of recommendation and is not to be considered as a HIV intervention that should be recommended for MSM. However, many MSM in Europe use this tactic and so communication around serosorting, including the risk of HIV transmission and acquisition as well as STI acquisition, is important to address in counselling and information to MSM.

Encouragingly, fifteen interventions were graded to be strongly, probably or possibly recommended. These interventions can complement each other to maximise their impact and to address prevention needs and preferences of a diverse population of MSM. Offering and implementing prevention packages in collaboration with community members is crucial to the success of national and sub-national prevention programmes in the EU/EEA. In the relatively resource-rich European setting, there is an opportunity to provide global leadership with regard to the regional scale-up of comprehensive effective HIV prevention interventions for MSM.

\section{Acknowledgements}

This research is part of a project commissioned by the European Centre for Disease Prevention and Control (ECDC) under contract ECDC/2012/043.

Conflict of interest

Authors report no conflict of interest.

\section{Authors' contributions}

SS prepared the manuscript and managed revisions. MS and SS carried out the data collection. AT led the study team. All authors participated in the study design, the interpretation of data and revised the manuscript for intellectual content. All authors approved the final manuscript.

\section{References}

1. World Health Organization (WHO). Global Health Observatory. [Accessed 25 May 2014]. Geneva: WHO; 2014. Available from: http://www.who.int/gho/hiv/en/.

2. Joint United Nations Programme on HIV/AIDS (UNAIDS). Global Report: UNAIDS report on the global AIDS epidemic 2013. Geneva: UNAIDS; 2013. Available from: http:// www.unaids.org/sites/default/files/en/media/unaids/ contentassets/documents/epidemiology/2013/gr2013/ UNAIDS_Global_Report_2013_en.pdf

3. Beyrer C, Sullivan PS, Sanchez J, Dowdy D, Altman D, Trapence $G$, et al. A call to action for comprehensive HIV services for men who have sex with men. Lancet. 2012;380(9839):424 38. http://dx.doi.org/10.1016/S0140-6736(12)61022-8 PMID:22819663

4. Beyrer C, Baral SD, van Griensven F, Goodreau SM, Chariyalertsak S, Wirtz AL, et al. Global epidemiology 
of HIV infection in men who have sex with men. Lancet. 2012;380(9839):367-77. http://dx.doi.org/10.1016/S01406736(12)60821-6 PMID:22819660

5. Sullivan PS, Jones JS, Baral SD. The global north: HIV epidemiology in high-income countries. Curr Opin HIV AIDS. 2014;9(2):199-205. http://dx.doi.org/10.1097/ COH.0000000000000039 PMID:24445370

6. Sullivan PS, Carballo-Diéguez A, Coates T, Goodreau SM, McGowan I, Sanders EJ, et al. Successes and challenges of HIV prevention in men who have sex with men. Lancet. 2012;380(9839):388-99. http://dx.doi.org/10.1016/S01406736(12)60955-6 PMID:22819659

7. Mayer KH, Bekker LG, Stall R, Grulich AE, Colfax G, Lama JR. Comprehensive clinical care for men who have sex with men: an integrated approach. Lancet. 2012;380(9839):37887. http://dx.doi.org/10.1016/S0140-6736(12)60835-6 PMID:22819653

8. ECDC and WHO Regional Office for Europe. HIV Surveillance in Europe. ECDC Surveillance report. ECDC: Stockholm; 2013. Available from: http://ecdc.europa.eu/en/publications/ publications/20121130-annual-hiv-surveillance-report.pdf

9. European Centre for Disease Prevention and Control (ECDC). Sexually transmitted infections in Europe, 1990-2009. Stockholm; ECDC; 2011. Available from: http://ecdc.europa. eu/en/publications/publications/110526_sur_sti_in europe_1990-2009.pdf.

10. European Centre for Disease Prevention and Control (ECDC). Thematic report: Men who have sex with men. Monitoring implementation of the Dublin Declaration on Partnership to Fight HIV/AIDS in Europe and Central Asia: 2012 Progress Report. Stockholm: ECDC; 2013. ECDC.

11. Baggaley RF, White RG, Boily MC. HIV transmission risk through anal intercourse: systematic review, meta-analysis and implications for HIV prevention. Int J Epidemiol. 2010;39(4):1048-63. http://dx.doi.org/10.1093/ije/dyq057 PMID:20406794

12. Brenner BG, Roger M, Stephens D, Moisi D, Hardy I, Weinberg J, et al.; Montreal PHI Cohort Study Group. Transmission clustering drives the onward spread of the HIV epidemic among men who have sex with men in Quebec. J Infect Dis. 2011;204(7):1115-9. http://dx.doi.org/10.1093/infdis/jir468 PMID:21881127

13. Lewis F, Hughes GJ, Rambaut A, Pozniak A, Leigh Brown AJ. Episodic sexual transmission of HIV revealed by molecular phylodynamics. PLoS Med. 2008;5(3):e50. http://dx.doi. org/10.1371/journal.pmed.0050050 PMID:18351795

14. Chan PA, Kazi S, Rana A, Blazar I, Dejong CC, Mayer KH, et al. Short communication: new HIV infections at Southern New England academic institutions: implications for prevention. AIDS Res Hum Retroviruses. 2013;29(1):25-9. http://dx.doi. org/10.1089/aid.2012.0130 PMID:22724920

15. European Centre for Disease Prevention and Control (ECDC). A comprehensive approach to HIV/STI prevention in the context of sexual health in the EU/EEA. Stockholm: ECDC, 2013.

16. Janiec J, Haar K, Spiteri G, Likatavicius G, Van de Laar M, Amato-Gauci AJ. Surveillance of human immunodeficiency virus suggests that younger men who have sex with men are at higher risk of infection, European Union, 2003 to 2012. Euro Surveill. 2013;18(48):20644. http://dx.doi.org/10.2807/15607917.ES2013.18.48.20644 PMID:24308979

17. World Health Organization. Prevention and treatment of HIV and other sexually transmitted infection among men who have sex with men and transgender people: Annexes. World Health Organization, Geneva, 2011. Available from: http://whqlibdoc. who.int/publications/2011/9789241501750_eng_annexes.pdf.

18. Berg R. The effectiveness of behavioural and psychosocial HIV/STI prevention interventions for MSM in Europe: a systematic review. Eurosurveillance 2009; 14(48):7

19. Beyrer W, Wirtz A, Walker D, Johns B, Sifakis F, Baral S. The Global HIV Epidemics among Men who have Sex with Men. Baltimore: Johns Hopkins Bloomberg School of Public Health; 2011.

20. Baral SD, Wirtz A, Sifakis F, Johns B, Walker D, Beyrer C. The highest attainable standard of evidence (HASTE) for HIV/AIDS interventions: toward a public health approach to defining evidence. Public Health Rep. 2012;127(6):572-84. Epub20121102. PMID:23115382

21. ECDC. A comprehensive approach to HIV and STI prevention among men who have sex with men. Stockholm: ECDC. In press 2015.

22. Baral SD, Poteat T, Strömdahl S, Wirtz AL, Guadamuz TE, Beyrer C. Worldwide burden of HIV in transgender women: a systematic review and meta-analysis. Lancet Infect Dis. 2013;13(3):214-22. http://dx.doi.org/10.1016/S14733099(12)70315-8 PMID:23260128
23. European Centre for Disease Prevention and Control (ECDC). Sexually Transmitted infections in Europe 1990-2010. Stockholm: ECDC; 2011. Available from: http://ecdc.europa.eu/ en/publications/Publications/201206-Sexually-TransmittedInfections-Europe-2010.pdf

24. Schünemann H, Hill S, Guyatt G, Akl EA, Ahmed F. The GRADE approach and Bradford Hill's criteria for causation. J Epidemiol Community Health. 2011;65(5):392-5. http://dx.doi. org/10.1136/jech.2010.119933 PMID:20947872

25. Sackett DL, Straus SE, Richardson WS, Rosenberg W, Haynes RB. Evidence based medicine: how to practice and teach EBM. New York: Churchill Livingstone; 2000.

26. Darzins PJ, Smith BJ, Heller RF. How to read a journal article. Med J Aust. 1992;157(6):389-94. Epub19920921. PMID:1447989</jrn〉

27. World Health Organization (WHO). Prevention and treatment of HIV and other sexually transmitted infections among men who have sex with men and transgender people: recommendations for a public health approach 2011. Geneva: WHO; 2011. Available from: http://www.who.int/hiv/pub/guidelines/ msm guidelines2011/en/.

28. Detels R, English P, Visscher BR, Jacobson L, Kingsley LA, Chmiel JS, et al. Seroconversion, sexual activity, and condom use among 2915 HIV seronegative men followed for up to 2 years. J Acquir Immune Defic Syndr. 1989;2(1):77-83. Epub19890101. PMID:2918462

29. Difranceisco W, Ostrow DG, Chmiel JS. Sexual adventurism, high-risk behavior, and human immunodeficiency virus-1 seroconversion among the Chicago MACS-CCS cohort, 1984 to 1992. A case-control study. Sex Transm Dis. 1996;23(6):4536o. http://dx.doi.org/10.1097/00007435-199611000-00003 PMID:8946628

30. Jin F, Crawford J, Prestage GP, Zablotska I, Imrie J, Kippax SC, et al. Unprotected anal intercourse, risk reduction behaviours, and subsequent HIV infection in a cohort of homosexual men. AIDS. 2009;23(2):243-52. http://dx.doi.org/10.1097/ QAD.ob013e32831fb51a PMID:19098494

31. Marks G, Millett GA, Bingham T, Lauby J, Murrill CS, Stueve A. Prevalence and protective value of serosorting and strategic positioning among Black and Latino men who have sex with men. Sex Transm Dis. 2010;37(5):325-7. Epub20100119. PMID:20081556

32. Golden MR, Stekler J, Hughes JP, Wood RW. HIV serosorting in men who have sex with men: is it safe? I Acquir Immune Defic Syndr. 2008;49(2):212-8. http://dx.doi.org/10.1097/ QAI.ob013e31818455e8 PMID:18769346

33. Berg R. The effectiveness of behavioural and psychosocial HIV/STI prevention interventions for MSM in Europe: A systematic review. Euro Surveill. 2009;14(48). Epub20091217. PMID:20003895

34. European Centre for Disease Prevention and Control (ECDC). Sexually transmitted infections in Europe 2011. Stockholm: ECDC; 2013. Available from: http://ecdc.europa.eu/en/ publications/Publications/sexually-transmitted-infectionsEurope-2011.pdf

35. Schmidt AJ, Benvenuti S, Breveglieri M. The European MSM internet survey (EMIS) UNGASS indicators. 2010. Available from: http://www.emis-project.eu/sites/default/files/public/ publications/EMIS_UNGASS_eng.pdf

36. EMIS. The European MSM Internet Survey. Available from: http://www.emis-project.eu

37. Cohen MS, Chen YQ, McCauley M, Gamble T, Hosseinipour MC, Kumarasamy N, et al.; HPTN 052 Study Team. Prevention of HIV-1 infection with early antiretroviral therapy. N Engl J Med. 2011;365(6):493-505. http://dx.doi.org/10.1056/ NEJMoa1105243 PMID:21767103

38. Lorimer K, Kidd L, Lawrence M, McPherson K, Cayless S, Cornish F. Systematic review of reviews of behavioural HIV prevention interventions among men who have sex with men. AIDS Care. 2013;25(2):133-50. http://dx.doi.org/10.1080/09540 121.2012.699672 PMID:22774763

39. Herbst JH, Sherba RT, Crepaz N, Deluca JB, Zohrabyan L, Stall RD, et al.; HIV/AIDS Prevention Research Synthesis Team. A meta-analytic review of HIV behavioral interventions for reducing sexual risk behavior of men who have sex with men. J Acquir Immune Defic Syndr. 2005;39(2):228-41. Epub20050521. PMID:15905741

40. Herbst JH, Beeker C, Mathew A, McNally T, Passin WF, Kay LS, et al.; Task Force on Community Preventive Services. The effectiveness of individual-, group-, and community-level HIV behavioral risk-reduction interventions for adult men who have sex with men: a systematic review. Am J Prev Med. 2007;32(4) Suppl;S38-67. http://dx.doi.org/10.1016/j.amepre.2006.12.006 PMID:17386336

41. Berg R. The effectiveness of behavioural and psychosocial HIV/ STI prevention interventions for MSM in Europe: A systematic 
review. Euro Surveill. 2009;14(48):19430. Epub20091217. PMID:20003895

42. McCusker J, Stoddard AM, Mayer KH, Zapka J, Morrison C, Saltzman SP. Effects of HIV antibody test knowledge on subsequent sexual behaviors in a cohort of homosexually active men. Am J Public Health. 1988;78(4):462-7. http:// dx.doi.org/10.2105/AJPH.78.4.462 PMID:3162357

43. Valleroy LA, MacKellar DA, Karon JM, Rosen DH, McFarland W, Shehan DA, et al.Young Men's Survey Study Group. HIV prevalence and associated risks in young men who have sex with men. JAMA. 2000;284(2):198-204. http://dx.doi. org/10.1001/jama.284.2.198 PMID:10889593

44. Colfax GN, Buchbinder SP, Cornelisse PG, Vittinghoff E, Mayer K, Celum C. Sexual risk behaviors and implications for secondary HIV transmission during and after HIV seroconversion. AIDS. 2002;16(11):1529-35. http://dx.doi. org/10.1097/00002030-200207260-00010 PMID:12131191

45. Marks G, Crepaz N, Senterfitt JW, Janssen RS. Meta-analysis of high-risk sexual behavior in persons aware and unaware they are infected with HIV in the United States: implications for HIV prevention programs. J Acquir Immune Defic Syndr. 2005;39(4):446-53. http://dx.doi.org/10.1097/01. qai.0000151079.33935.79 PMID:16010168

46. Rogstad K, Palfreeman A, Rooney G, Hart GJ, Lowbury R, Mortimer P, et al.; Clinical Effectiveness Group, British Association of Sexual Health and HIV. UK National Guidelines on HIV Testing 2006. Int J STD AIDS. 2006;17(10):66876. http://dx.doi.org/10.1258/095646206780071045 PMID:17059636

47. Scott J, Bansi L, Ivens D. HIV test uptake after introducing an opt-out screening system. Int J STD AIDS. 2006;17(3):213. http://dx.doi.org/10.1258/095646206775809277 PMID:16510018

48. National AIDS Trust. Updating our strategies: report of an expert seminar on HIV tesing and prevention. London: National AIDS Trust; 2007. Available from: http://www.nat.org.uk/ Media\%2olibrary/Files/PDF\%2odocuments/NAT-Updating-ourstrategies.pdf

49. Golombok S, Harding R, Sheldon J. An evaluation of a thicker versus a standard condom with gay men. AIDS. 2001;15(2):24550. http://dx.doi.org/10.1097/00002030-200101260-00015 PMID:11216934

50. Voeller B, Coulson AH, Bernstein GS, Nakamura RM. Mineral oil lubricants cause rapid deterioration of latex condoms. Contraception. 1989;39(1):95-102. http://dx.doi. org/10.1016/0010-7824(89)90018-8 PMID:2535978

51. White N, Taylor K, Lyszkowski A, Tullett J, Morris C. Dangers of lubricants used with condoms. Nature. 1988;335(6185):19. http://dx.doi.org/10.1038/335019ao PMID:3412452

52. Smith AM, Jolley D, Hocking J, Benton K, Gerofi J. Does penis size influence condom slippage and breakage? Int J STD AIDS. 1998;9(8):444-7. http://dx.doi.org/10.1258/0956462981922593 PMID:9702591

53. Smith AM, Jolley D, Hocking J, Benton K, Gerofi J. Does additional lubrication affect condom slippage and breakage? Int J STD AIDS. 1998;9(6):330-5. http://dx.doi. org/10.1258/0956462981922359 PMID:9671246

54. Carballo-Diéguez A, Stein Z, Sáez H, Dolezal C, Nieves-Rosa $L$, Díaz F. Frequent use of lubricants for anal sex among men who have sex with men: the HIV prevention potential of a microbicidal gel. Am J Public Health. 2000;90(7):1117-21. http://dx.doi.org/10.2105/AJPH.90.7.1117 PMID:1089719

55. Baral S, Trapence G, Motimedi F, Umar E, lipinge S, Dausab F, et al. HIV prevalence, risks for HIV infection, and human rights among men who have sex with men (MSM) in Malawi, Namibia, and Botswana. PLoS ONE. 2009;4(3):e4997. http://dx.doi. org/10.1371/journal.pone.0004997 PMID:19325707

56. Butler LM, Osmond DH, Jones AG, Martin JN. Use of saliva as a lubricant in anal sexual practices among homosexual men. J Acquir Immune Defic Syndr. 2009;50(2):162-7. http://dx.doi. org/10.1097/QAI.ob013e31819388a9 PMID:19131893

57. Lunding S, Katzenstein TL, Kronborg G, Lindberg JA, Jensen J, Nielsen $\mathrm{HI}$, et al. The Danish PEP registry: experience with the use of postexposure prophylaxis (PEP) following sexual exposure to HIV from 1998 to 2006. Sex Transm Dis. 2010;37(1):49-52. http://dx.doi.org/10.1097/ OLQ.ob013e3181b6f284 PMID:19734819

58. Sonder GJ, van den Hoek A, Regez RM, Brinkman K, Prins JM, Mulder JW, et al. Trends in HIV postexposure prophylaxis prescription and compliance after sexual exposure in Amsterdam, 2000-2004. Sex Transm Dis. 2007;34(5):288-93. Epub20060919. PMID:16980918

59. Richardson JL, Milam J, McCutchan A, Stoyanoff S, Bolan R, Weiss J, et al. Effect of brief safer-sex counseling by medical providers to HIV-1 seropositive patients: a multi-clinic assessment. AIDS. 2004;18(8):1179-86. http://dx.doi. org/10.1097/00002030-200405210-00011 PMID:15166533

6o. McKirnan DJ, Tolou-Shams M, Courtenay-Quirk C. The Treatment Advocacy Program: a randomized controlled trial of a peer-led safer sex intervention for HIV-infected men who have sex with men. J Consult Clin Psychol. 2010;78(6):952-63. http://dx.doi.org/10.1037/a0020759 PMID:20919760

61. Safren SA, O’Cleirigh CM, Skeer M, Elsesser SA, Mayer $\mathrm{KH}$. Project enhance: a randomized controlled trial of an individualized HIV prevention intervention for HIV-infected men who have sex with men conducted in a primary care setting. Health Psychol. 2013;32(2):171-9. http://dx.doi.org/10.1037/ a0028581 PMID:22746262

62. Buchacz K, Patel P, Taylor M, Kerndt PR, Byers RH, Holmberg SD, et al. Syphilis increases HIV viral load and decreases CD4 cell counts in HIV-infected patients with new syphilis infections. AIDS. 2004;18(15):2075-9. http://dx.doi. org/10.1097/00002030-200410210-00012 PMID:15577629

63. Johnson WD, Diaz RM, Flanders WD, Goodman M, Hill AN, Holtgrave D, et al. Behavioral interventions to reduce risk for sexual transmission of HIV among men who have sex with men. Cochrane Database Syst Rev. 2008; (3):CDo01230. Epub20080723. PMID:18646068

64. Kalichman SC, Rompa D, Cage M, DiFonzo K, Simpson D, Austin J, et al. Effectiveness of an intervention to reduce HIV transmission risks in HIV-positive people. Am J Prev Med. 2001;21(2):84-92. http://dx.doi.org/10.1016/S07493797(01)00324-5 PMID:11457627

65. Rosser BR, Oakes JM, Konstan J, Hooper S, Horvath KJ, Danilenko GP, et al. Reducing HIV risk behavior of men who have sex with men through persuasive computing: results of the Men's INTernet Study-II. AIDS. 2010;24(13):2099107. http://dx.doi.org/10.1097/QAD.ob013e32833C4ac7 PMID:20601853

66. Hirshfield $\mathrm{S}$, Chiasson MA, Joseph $H$, Scheinmann $R$, Johnson WD, Remien $\mathrm{RH}$, et al. An online randomized controlled trial evaluating HIV prevention digital media interventions for men who have sex with men. PLoS ONE. 2012;7(10):e46252. http:// dx.doi.org/10.1371/journal.pone.0046252 PMID:23071551

67. Lau JT, Lau M, Cheung A, Tsui HY. A randomized controlled study to evaluate the efficacy of an Internet-based intervention in reducing HIV risk behaviors among men who have sex with men in Hong Kong. AIDS Care. 2008;20(7):820-8. http://dx.doi. org/10.1080/09540120701694048 PMID:18608057

68. Carpenter KM, Stoner SA, Mikko AN, Dhanak LP, Parsons JT. Efficacy of a web-based intervention to reduce sexual risk in men who have sex with men. AIDS Behav. 2010;14(3):549-57. http://dx.doi.org/10.1007/s10461-009-9578-2 PMID:19499321

69. Simon Rosser BR, West W, Weinmeyer R. Are gay communities dying or just in transition? Results from an international consultation examining possible structural change in gay communities. AIDS Care. 2008;20(5):588-95. http://dx.doi. org/10.1080/09540120701867156 PMID:18484330

70. Liau A, Millett G, Marks G. Meta-analytic examination of online sex-seeking and sexual risk behavior among men who have sex with men. Sex Transm Dis. 2006;33(9):576-84. http://dx.doi. org/10.1097/01.olq.0000204710.35332.C5 PMID:16540884

71. McFarlane M, Bull SS, Rietmeijer CA. The Internet as a newly emerging risk environment for sexually transmitted diseases. JAMA. 2000;284(4):443-6. http://dx.doi.org/10.1001/ jama.284.4.443 PMID:10904506

72. Lewnard JA, Berrang-Ford L. Internet-based partner selection and risk for unprotected anal intercourse in sexual encounters among men who have sex with men: a meta-analysis of observational studies. Sex Transm Infect. 2014;90(4):2906. http://dx.doi.org/10.1136/sextrans-2013-051332 PMID:24518249

73. Huebner DM, Binson D, Woods WJ, Dilworth SE, Neilands TB, Grinstead O. Bathhouse-based voluntary counseling and testing is feasible and shows preliminary evidence of effectiveness. I Acquir Immune Defic Syndr. 2006;43(2):239 46. http://dx.doi.org/10.1097/01.qai.0000242464.50947.16 PMID:16951645

74. Daskalakis D, Silvera R, Bernstein K, Stein D, Hagerty R, Hutt R, et al. Implementation of HIV testing at 2 New York City bathhouses: from pilot to clinical service. Clin Infect Dis. 2009;48(11):1609-16. http://dx.doi.org/10.1086/598979 PMID:19400690

75. Spielberg F, Branson BM, Goldbaum GM, Kurth A, Wood RW. Designing an HIV counseling and testing program for bathhouses: the Seattle experience with strategies to improve acceptability. J Homosex. 2003;44(3-4):203-20. http://dx.doi. org/10.1300/Jo82V44n03_09 PMID:12962183

76. Mullens AB, Staunton S, Debattista J, Hamernik E, Gill D. Sex on premises venue (SOPV) health promotion project in response to sustained increases in HIV notifications. Sex 
Health. 2009;6(1):41-4. http://dx.doi.org/10.1071/SHo7087 PMID:19254490

77. Raymond HF, Bingham T, McFarland W. Locating unrecognized HIV infections among men who have sex with men: San Francisco and Los Angeles. AIDS Educ Prev. 2008;20(5):408-19. http://dx.doi.org/10.1521/aeap.2008.20.5.408 PMID:18956982

78. Wei C, Herrick A, Raymond HF, Anglemyer A, Gerbase A, Noar SM. Social marketing interventions to increase HIV/STI testing uptake among men who have sex with men and male-to-female transgender women. Cochrane Database Syst Rev. 2011; (9):CDo09337. Epub20110909. PMID:21901734

79. Silvestre AJ, Hylton JB, Johnson LM, Houston C, Witt M, Jacobson $L$, et al. Recruiting minority men who have sex with men for HIV research: results from a 4-city campaign. Am Public Health. 2006;96(6):1020-7. http://dx.doi.org/10.2105/ AJPH.2005.072801 PMID:16670218

80. Darrow WW, Biersteker S. Short-term impact evaluation of a social marketing campaign to prevent syphilis among men who have sex with men. Am J Public Health. 2008;98(2):337-43. http://dx.doi.org/10.2105/AJPH.2006.109413 PMID:18172146

81. Pedrana A, Hellard M, Guy R, El-Hayek C, Gouillou M, Asselin J, et al. Stop the drama Downunder: a social marketing campaign increases HIV/sexually transmitted infection knowledge and testing in Australian gay men. Sex Transm Dis. 2012;39(8):6518. http://dx.doi.org/10.1097/OLQ.ob013e318255dfo6 PMID:22801349

82. Martínez-Donate AP, Zellner JA, Sañudo F, Fernandez-Cerdeño A, Hovell MF, Sipan CL, et al. Hombres Sanos: evaluation of a social marketing campaign for heterosexually identified Latino men who have sex with men and women. Am J Public Health. 2010;100(12):2532-40. http://dx.doi.org/10.2105/ AJPH.2009.179648 PMID:21068423

83. Grant RM, Lama JR, Anderson PL, McMahan V, Liu AY, Vargas L, et al.; iPrEx Study Team. Preexposure chemoprophylaxis for HIV prevention in men who have sex with men. $\mathrm{N} \mathrm{Engl}$ J Med. 2010;363(27):2587-99. http://dx.doi.org/10.1056/ NEJMoa1011205 PMID:21091279

84. Wiysonge CS, Kongnyuy EJ, Shey M, Muula AS, Navti OB, Akl $E A$, et al. Male circumcision for prevention of homosexual acquisition of HIV in men. Cochrane Database Syst Rev. 2011; (6):CDo07496. Epub20110617. PMID:21678366

85. Sánchez J, Sal Y Rosas VG, Hughes JP, Baeten JM, Fuchs J, Buchbinder SP, et al. Male circumcision and risk of HIV acquisition among MSM. AIDS. 2011;25(4):519-23. http:// dx.doi.org/10.1097/QAD.ob013e328340fd81 PMID:21099672

86. Schwappach DL, Bruggmann P. An integrated model of care to counter high incidence of HIV and sexually transmitted diseases in men who have sex with men - initial analysis of service utilizers in Zurich. BMC Public Health. 2008;8(1):180. http://dx.doi.org/10.1186/1471-2458-8-180 PMID:18505556

87. Newman CE, Kippax SC, Mao L, Rogers GD, Saltman DC, Kidd $M R$. Features of the management of depression in gay men and men with HIV from the perspective of Australian GPs. Fam Pract. 2009;26(1):27-33. http://dx.doi.org/10.1093/fampra/ cmno89 PMID:19011172

88. Down I, Wilson DP, McCann PD, Gray R, Hoare A, Bradley , et al. Increasing gay men's testing rates and enhancing partner notification can reduce the incidence of syphilis. Sex Health. 2012;9(5):472-80. http://dx.doi.org/10.1071/SH12023 PMID:23380198

89. Woodward CL, Roedling S, Edwards SG, Armstrong A, Richens J. Computer-assisted survey of attitudes to HIV and sexually transmissible infection partner notification in HIV-positive men who have sex with men. Sex Health. 2010;7(4):460-2. http:// dx.doi.org/10.1071/SHo9146 PMID:21062587

90. Ehrhardt AA, Sawires S, McGovern T, Peacock D, Weston M. Gender, empowerment, and health: what is it? How does it work? J Acquir Immune Defic Syndr. 2009;51(Suppl 3):S96105. http://dx.doi.org/10.1097/QAl.obo13e3181aafd54 PMID:19553784

91. Peacock D, Stemple L, Sawires S, Coates TJ. Men, HIV/AIDS, and human rights. J Acquir Immune Defic Syndr. 2009;51(Suppl 3):S119-25. http://dx.doi.org/10.1097/QAl.ob013e3181aafd8a PMID:19553779

92. Renzi C, Tabet SR, Stucky JA, Eaton N, Coletti AS, Surawicz CM, et al. Safety and acceptability of the Reality condom for anal sex among men who have sex with men. AIDS. 2003;17(5):72731. http://dx.doi.org/10.1097/00002030-200303280-00011 PMID:12646796

93. Jin F, Prestage GP, Templeton DJ, Poynten IM, Donovan B, Zablotska I, et al. The impact of HIV seroadaptive behaviors on sexually transmissible infections in HIV-negative homosexual men in Sydney, Australia. Sex Transm Dis. 2012;39(3):1914. http://dx.doi.org/10.1097/OLQ.obo13e3182401a2 PMID:22337105
94. Philip SS, Yu X, Donnell D, Vittinghoff E, Buchbinder S. Serosorting is associated with a decreased risk of HIV seroconversion in the EXPLORE Study Cohort. PLoS ONE. 2010;5(9):e12662. http://dx.doi.org/10.1371/journal. pone.0012662 PMID:20844744

95. McDaid LM, Hart GJ. Serosorting and strategic positioning during unprotected anal intercourse: are risk reduction strategies being employed by gay and bisexual men in Scotland? Sex Transm Dis. 2012;39(9):735-8. http://dx.doi. org/10.1097/OLQ.ob013e31825a3a3C PMID:22902673

96. Dubois-Arber F, Jeannin A, Lociciro S, Balthasar H. Risk reduction practices in men who have sex with men in Switzerland: serosorting, strategic positioning, and withdrawal before ejaculation. Arch Sex Behav. 2012;41(5):1263-72. http:// dx.doi.org/10.1007/s10508-011-9868-4 PMID:22083656

97. Politch JA, Mayer KH, Welles SL, O’Brien WX, Xu C, Bowman FP, et al. Highly active antiretroviral therapy does not completely suppress HIV in semen of sexually active HIV-infected men who have sex with men. AIDS. 2012;26(12):1535-43. http://dx.doi. org/10.1097/QAD.obo13e328353b11b PMID:22441253

98. Casper C, Wald A, Pauk J, Tabet SR, Corey L, Celum CL. Correlates of prevalent and incident Kaposi's sarcomaassociated herpesvirus infection in men who have sex with men. J Infect Dis. 2002;185(7):990-3. http://dx.doi. org/10.1086/339605 PMID:11920325

99. Soderberg LS. Immunomodulation by nitrite inhalants may predispose abusers to AIDS and Kaposi's sarcoma. Jeuroimmunol. 1998;83(1-2):157-61. http://dx.doi. org/10.1016/S0165-5728(97)00232-4 PMID:9610684

100. Dax EM, Adler WH, Nagel JE, Lange WR, Jaffe JH. Amyl nitrite alters human in vitro immune function. Immunopharmacol Immunotoxicol. 1991;13(4):577-87. http://dx.doi. org/10.3109/08923979109019724 PMID:1685501

101. van Griensven GJ, Tielman RA, Goudsmit J, van der Noordaa J, de Wolf F, de Vroome EM, et al. Risk factors and prevalence of HIV antibodies in homosexual men in the Netherlands. Am J Epidemiol. 1987;125(6):1048-57. Epub19870601. PMID:3495173

102. Ostrow DG, DiFranceisco WJ, Chmiel JS, Wagstaff DA, Wesch J. A case-control study of human immunodeficiency virus type 1 seroconversion and risk-related behaviors in the Chicago MACS/CCS Cohort, 1984-1992. Multicenter AIDS Cohort Study. Coping and Change Study. Am J Epidemiol. 1995;142(8):87583. Epub19951015. PMID:7572964

103. Chesney MA, Barrett DC, Stall R. Histories of substance use and risk behavior: precursors to HIV seroconversion in homosexual men. Am J Public Health. 1998;88(1):113-6. http:// dx.doi.org/10.2105/AJPH.88.1.113 PMID:9584015

104. Buchbinder SP, Vittinghoff E, Heagerty PJ, Celum CL, Seage GR 3rd, Judson FN, et al. Sexual risk, nitrite inhalant use, and lack of circumcision associated with HIV seroconversion in men who have sex with men in the United States. J Acquir Immune Defic Syndr. 2005;39(1):82-9. http://dx.doi.org/10.1097/01. qai.0000134740.41585.f4 PMID:15851918

105. Velasquez MM, von Sternberg K, Johnson DH, Green C, Carbonari JP, Parsons JT. Reducing sexual risk behaviors and alcohol use among HIV-positive men who have sex with men: a randomized clinical trial. J Consult Clin Psychol. 2009;77(4):657-67. http://dx.doi.org/10.1037/a0015519 PMID:19634959

106. Merson M, Padian N, Coates TJ, Gupta GR, Bertozzi SM, Piot $P$, et al.; Lancet HIV Prevention Series Authors. Combination HIV prevention. Lancet. 2008;372(9652):1805-6. http://dx.doi. org/10.1016/S0140-6736(08)61752-3 PMID:19027478

107. European Centre for Disease Prevention and Control (ECDC). HIV/AIDS Surveillance in Europe 2013. Stockholm: ECDC; 2014.

108. Brown AE, Gill ON, Delpech VC. HIV treatment as prevention among men who have sex with men in the UK: is transmission controlled by universal access to HIV treatment and care? HIV Med. 2013;14(9):563-70. http://dx.doi.org/10.1111/hiv.12066 PMID:23890150

109. Greacen T, Friboulet D, Fugon L, Hefez S, Lorente N, Spire $B$. Access to and use of unauthorised online HIV self-tests by internet-using French-speaking men who have sex with men. Sex Transm Infect. 2012;88(5):368-74. http://dx.doi. org/10.1136/sextrans-2011-050405 PMID:22436195

110.Lorente N, Fugon L, Carrieri MP, Andreo C, Le Gall JM, Cook $\mathrm{E}$, et al. Acceptability of an "on-demand" pre-exposure HIV prophylaxis trial among men who have sex with men living in France. AIDS Care. 2012;24(4):468-77. Epub20111117. PMID:22085083

111. Aghaizu A, Mercey D, Copas A, Johnson AM, Hart G, Nardone $A$. Who would use PrEP? Factors associated with intention to use among MSM in London: a community survey. Sex Transm Infect. 2012. Epub20120928. PMID:23015689

112. Solomon MM, Lama JR, Glidden DV, Mulligan K, McMahan V, Liu AY, et al.; iPrEx Study Team. Changes in renal 
function associated with oral emtricitabine/tenofovir disoproxil fumarate use for HIV pre-exposure prophylaxis. AIDS. 2014;28(6):851-9. http://dx.doi.org/10.1097/ QAD.0000000000000156 PMID:24499951

113. Grant RM, Anderson PL, McMahan V, Liu A, Amico KR, Mehrotra M, et al.; iPrEx study team. Uptake of pre-exposure prophylaxis, sexual practices, and HIV incidence in men and transgender women who have sex with men: a cohort study. Lancet Infect Dis. 2014;14(9):820-9. http://dx.doi.org/10.1016/ S1473-3099(14)70847-3 PMID:25065857

114. Marcus JL, Glidden DV, Mayer KH, Liu AY, Buchbinder SP, Amico $K R$, et al. No evidence of sexual risk compensation in the iPrEx trial of daily oral HIV preexposure prophylaxis. PLoS ONE. 2013;8(12):e81997. http://dx.doi.org/10.1371/journal. pone.0081997 PMID:24367497

115. McCormack S, Dunn D. Pragmatic Open-Label Randomised Trial of Preexposure Prophylaxis: The PROUD Study. CRO 2015; Seattle, Washington, 2015. Available from: http:// www.croiconference.org/sessions/pragmatic-open-labelrandomised-trial-preexposure-prophylaxis-proud-study

116.Agence de recherche ANRS (France Recherche Nord\&Sud Sida-HIV Hépatites). A drug taken at the time of sexual intercourse effectively reduces the risk of infection. 29 October 2014. Available from: http://www.anrs.fr/content/ download/6008/32756/file/Press\%2orelease\%2olPERGAYWEB.pdf

117. European Union Agency for Fundamental Rights. LGBT Survey 2012. Available from: http://fra.europa.eu/DVS/DVT/lgbt.php.

118. Weinhardt LS, Forsyth AD, Carey MP, Jaworski BC, Durant LE. Reliability and validity of self-report measures of HIV-related sexual behavior: progress since 1990 and recommendations for research and practice. Arch Sex Behav. 1998;27(2):155-80. http://dx.doi.org/10.1023/A:1018682530519 PMID:9562899

119. Jaccard J, McDonald R, Wan CK, Dittus PJ, Quinlan S. The Accuracy of Self-Reports of Condom Use and Sexual Behavior. J Appl Soc Psychol. 2002;32(9):1863-905. http://dx.doi. org/10.1111/j.1559-1816.2002.tboo263.x

120. Stone E, Heagerty P, Vittinghoff E, Douglas JM Jr, Koblin BA, Mayer $\mathrm{KH}$, et al. Correlates of condom failure in a sexually active cohort of men who have sex with men. J Acquir Immune Defic Syndr Hum Retrovirol. 1999;20(5):495-501. http://dx.doi. org/10.1097/00042560-199904150-00013 PMID:10225233

121. Del Romero J, Castilla J, Hernando V, Rodríguez C, García S. Combined antiretroviral treatment and heterosexual transmission of HIV-1: cross sectional and prospective cohort study. BMJ. 2010;340(may14 1):C2205. http://dx.doi. org/10.1136/bmj.c2205 PMID:20472675

122. Bunnell R, Ekwaru JP, Solberg P, Wamai N, Bikaako-Kajura $W$, Were W, et al. Changes in sexual behavior and risk of HIV transmission after antiretroviral therapy and prevention interventions in rural Uganda. AIDS. 2006;20(1):85-92. http://dx.doi.org/10.1097/01.aids.0000196566.40702.28 PMID:16327323

123. Donnell D, Baeten IM, Kiarie J, Thomas KK, Stevens W, Cohen CR, et al.; Partners in Prevention HSV/HIV Transmission Study Team. Heterosexual HIV-1 transmission after initiation of antiretroviral therapy: a prospective cohort analysis. Lancet. 2010;375(9731):2092-8. http://dx.doi.org/10.1016/S01406736(10)60705-2 PMID:20537376

124. Reynolds SJ, Makumbi F, Nakigozi G, Kagaayi J, Gray RH, Wawer M, et al. HIV-1 transmission among HIV-1 discordant couples before and after the introduction of antiretroviral therapy. AIDS. 2011;25(4):473-7. http://dx.doi.org/10.1097/ QAD.ob013e3283437C2b PMID:21160416

125. Marcus U, Hickson F, Weatherburn P, Schmidt AJ; EMIS Network. Prevalence of HIV among MSM in Europe: comparison of self-reported diagnoses from a large scale internet survey and existing national estimates. BMC Public Health. 2012;12(1):978. http://dx.doi.org/10.1186/1471-245812-978 PMID:23151263

126. Beyrer C, Wirtz AL, Walker D, Johns B, Sifakis F, Baral S. The global HIV epidemics among Men Who Have Sex With Men. The World Bank, D.C: 2011. Available from: http://siteresources.worldbank.org/INTHIVAIDS/ Resources/375798-1103037153392/MSMReport.pdf.

127.Grov C, Golub SA, Parsons JT. HIV status differences in venues where highly sexually active gay and bisexual men meet sex partners: results from a pilot study. AIDS Educ Prev. 2010;22(6):496-508. http://dx.doi.org/10.1521/ aeap.2010.22.6.496 PMID:21204626

128. Horvath KJ, Bowen AM, Williams ML. Virtual and physical venues as contexts for HIV risk among rural men who have sex with men. Health Psychol. 2006;25(2):237-42. http://dx.doi. org/10.1037/0278-6133.25.2.237 PMID:16569116

129. Millett GA, Flores SA, Marks G, Reed JB, Herbst JH. Circumcision status and risk of HIV and sexually transmitted infections among men who have sex with men: a metaanalysis. JAMA. 2008;300(14):1674-84. http://dx.doi. org/10.1001/jama.300.14.1674 PMID:18840841

130. Templeton DJ, Millett GA, Grulich AE. Male circumcision to reduce the risk of HIV and sexually transmitted infections among men who have sex with men. Curr Opin Infect Dis. 2010;23(1):45-52. http://dx.doi.org/10.1097/ QCO.obo13e328334e54d PMID:19935420

131.Jameson DR, Celum CL, Manhart L, Menza TW, Golden MR. The association between lack of circumcision and HIV, HSV-2, and other sexually transmitted infections among men who have sex with men. Sex Transm Dis. 2010;37(3):147-52. http:// dx.doi.org/10.1097/OLQ.ob013e3181bdoffo PMID:19901865

132. McDaid LM, Weiss HA, Hart GJ. Circumcision among men who have sex with men in Scotland: limited potential for HIV prevention. Sex Transm Infect. 2010;86(5):404-6. http:// dx.doi.org/10.1136/sti.2010.042895 PMID:20595141

133. Safeguarding male circumcision. Lancet. 2012;380(9845):860.

134. Wellings K, Collumbien M, Slaymaker E, Singh S, Hodges Z, Patel D, et al. Sexual behaviour in context: a global perspective. Lancet. 2006;368(9548):1706-28. http://dx.doi. org/10.1016/S0140-6736(06)69479-8 PMID:17098090

135. Rothenberg RB, Scarlett M, del Rio C, Reznik D, O’Daniels C. Oral transmission of HIV. AIDS. 1998;12(16):2095-105. http://dx.doi.org/10.1097/00002030-199816000-00004 PMID:9833850

136. Gilbart VL, Evans BG, Dougan S. HIV transmission among men who have sex with men through oral sex. Sex Transm Infect. 2004;80(4):324. http://dx.doi.org/10.1136/sti.2004.009217 PMID:15295136

137. Richters J, Grulich A, Ellard J, Hendry O, Kippax S. HIV transmission among gay men through oral sex and other uncommon routes: case series of HIV seroconverters, Sydney. AIDS. 2003;17(15):2269-71. http://dx.doi. org/10.1097/00002030-200310170-00020 PMID:14523289

138. Vosburgh HW, Mansergh G, Sullivan PS, Purcell DW. A review of the literature on event-level substance use and sexual risk behavior among men who have sex with men. AIDS Behav. 2012;16(6):1394-410. http://dx.doi.org/10.1007/s10461-0110131-8 PMID:22323004 